\title{
Magnetic Material Characterization Using an Inverse Problem Approach
}

\author{
Ahmed Abouelyazied Abdallh and Luc Dupré \\ Department of Electrical Energy, Systems and Automation, Ghent University \\ Belgium
}

\section{Introduction}

Soft magnetic materials are present in many electromagnetic devices and are widely used in industrial applications. In order to analyze magnetically such applications, e.g. by numerical techniques, the magnetic characteristics of the material inside the device have to be known.

There are several standard measurement techniques for characterizing magnetic materials, e.g. an Epstein frame is commonly used for identifying the magnetic properties of a material (Sievert, 2000). However, this requires extra samples of the magnetic material used in the electromagnetic device. These extra samples are often not available. Moreover, the characteristics of the magnetic material may be altered during the device manufacturing (Takahashi et al., 2008). Therefore, it is convenient to characterize the material on the specific geometry of the device itself.

In practice, the direct identification of the magnetic material exclusively based on magnetic measurements is quite difficult and sometimes impossible, due to the complexity of the electromagnetic device geometry. Therefore, an alternative method is needed. Recently, the coupled experimental-numerical inverse problem has gained a lot of interests for the identification problems (Tarantola, 2004).

The goal of this chapter is to design and develop a combined experimental-numerical algorithm for the magnetic characterization of the material, present in a geometrically complex structure, using an electromagnetic inverse problem approach. Specifically, we aim at identifying the single-valued $B-H$ curve, hysteresis characteristics, and loss parameters of a magnetic material inside an electromagnetic device.

The proposed coupled experimental-numerical inverse algorithm mainly consists of two major parts: experimental measurements and numerical modeling of the device. The proposed algorithm in this chapter is robust because all uncertainties present in the two parts are taken into account in a stochastic framework.

Standard techniques for magnetic material characterization is presented below, followed by the state-of-the-art of the proposed scheme. The proposed algorithm is validated by applying it for the identification of the magnetic properties of the material in an electromagnetic device, an EI electromagnetic core inductor. However, the proposed algorithm can be applied for any other application. 


\section{Standard techniques for magnetic material characterization}

Measurements are widely used when the measurement standards are fixed. Basic magnetic measurements in magnetic materials have been collected in a group of standards denoted by IEC 60404. The two main commonly used techniques to measure the global magnetic properties of sheets of magnetic materials are Epstein frame (IEC404-2, 1996) and single sheet tester (IEC404-3, 2000), which are considered as closed magnetic circuit and the magnetic path length inside the sample can be easily defined.

In section 2.1, we include the general principles for magnetic field and magnetic induction calculations in a closed magnetic circuit. The magnetic material characterization fully based on magnetic measurements are shown in section 2.2.

\subsection{General principles for magnetic field and induction evaluation in a closed magnetic circuit}

Consider a simple closed magnetic core, i.e. no air gap, consisting of a current carrying coil of $N_{1}$ turns and a magnetic core with a magnetic mean path length $l_{c}$ and a cross sectional area $A_{c}$ perpendicular to the field lines, as shown in Fig. 1(a). Assume that the relative magnetic permeability of the core material $\mu_{r}$ is very high so that all magnetic flux lines are confined within the core ${ }^{1}$.

The magnetic field strength at the surface of the core $H_{s}$ and the magnetic induction $B_{a v}$ inside the core material can be calculated using Ampere's law and Faraday's law, respectively.

Ampere's law in the integral form states that the total electric current $N_{1} i$ through the surface $S$ bounded by the specific closed contour $C$ induces the magnetomotive force (m.m.f.) in this contour:

$$
\oint_{C} \mathbf{H} \cdot d l=\oint_{S} \mathbf{J} \cdot d s
$$

with $\mathbf{J}$ being the total current density confined by the closed contour $C$. Hence, as a first approximation:

$$
H_{s} l_{c}=N_{1} i \quad \therefore \quad H_{s}=\frac{N_{1} i}{l_{c}}
$$

where $H_{S}$ is the magnetic field strength at the surface of the core, and $N_{1} i$ the magnetomotive force.

Consider a ferromagnetic material uniformly magnetized along one of its symmetry lines using a excitation windings $N_{1}$, as shown in Fig. 1(a). The sample is subjected to a time dependent magnetic field $H$. The flux density can be derived from the induced voltage in the measurement winding 'pickup coil' by the time varying total magnetic flux, according to Faraday's law:

$$
\begin{gathered}
V_{s e c}(t)=N_{2} \frac{d \phi}{d t}=N_{2} A_{c} \frac{d B_{a v}}{d t} \\
B_{a v}(t)=\frac{1}{N_{2} A_{c}} \int_{0}^{t} V_{s e c}(\tau) d \tau+B_{a v}(0)=\frac{1}{N_{2} A_{c}} \sum_{i=1}^{T / \Delta \tau} V_{\text {sec }_{i}} \Delta \tau+B_{a v}(0)
\end{gathered}
$$

\footnotetext{
${ }^{1}$ Indeed, in order to obtain homogenous magnetic field distributions in the magnetic sample, the excitation winding should be wound around the hole circumference of the magnetic sample.
} 


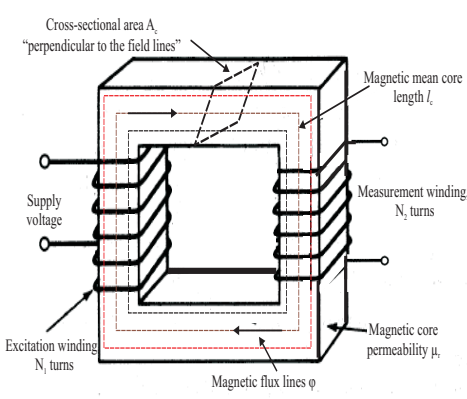

(a)

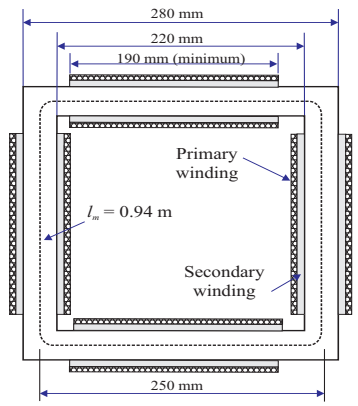

(b)

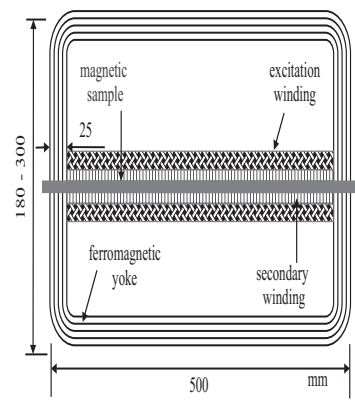

(c)

Fig. 1. (a) Schematic diagram of a simple magnetic circuit, (b) The standard Epstein frame according to IEC 60404-2 (IEC404-2, 1996), (c) The standard single sheet tester profile (IEC404-3, 2000).

where $N_{2}$ is the number of turns of the measurement winding, $T$ is the period of the secondary voltage, $B_{a v}(t)$ is the average flux density in the cross-section $A_{c}$ and $\Delta \tau$ is the sampling period. Whether the magnetic flux density is uniformly distributed over the cross section of the sample or not, the sensing coil measures the average induction value over the cross section $A_{c}{ }^{2}$.

\subsection{Magnetic material characterization fully based on magnetic measurements}

Figs. 1(b) and (c) show respectively the schematic diagram of the standard Epstein frame (IEC404-2, 1996) and single sheet tester (IEC404-3, 2000), which are considered as closed magnetic circuits. The $B-H$ characterization of the material with these techniques are possible, only based on electromagnetic measurements as shown in section 2.1, under the conditions that the magnetic field pattern is homogeneous and known in advance. Moreover the measurements are standardized resulting in geometrical parameters that have a fixed known value. However, this requires extra samples of the magnetic material used in the electromagnetic device. These extra samples are often not available. Therefore, it is convenient to characterize the material on the specific geometry of the device itself.

Alternatively, the local magnetic measurements can be used for the identification of the magnetic material properties on the specific geometry of the device itself. The local magnetic measurements consist of measuring two magnetic quantities, i.e. $H$ and $B$, locally at the same position. Several magnetic sensors are used for local magnetic measurements, such as the flat $H$-coil, fluxgate sensor, and the Hall-probe sensor 'for local magnetic field measurements', and the needle probes 'for local magnetic induction measurements', (Abdallh \& Dupré, 2010c). In practice, the local magnetic measurements are useful and accurate enough in the case that the local place is accessible and the magnetic field pattern is sufficiently homogeneous at that

\footnotetext{
${ }^{2}$ In order to minimize the measurement of any flux in the air, the measurement winding should be wound as close as possible to the magnetic sample. In practice, since the diameter of the measurement winding coil is thinner than the diameter of the excitation winding coil, the measurement winding is wound closer to the magnetic sample beneath the excitation winding.
} 
local position ${ }^{3}$. However, most of electromagnetic devices exhibit such non-uniform magnetic field patterns.

Therefore, we present in this chapter the inverse procedure for the identification of the magnetic properties of a magnetic material by interpreting well-defined measurements into a numerical model of the considered electromagnetic device.

\section{State-of-the-art of the proposed methodology}

The magnetic properties of a material can be recovered by the interpretation of well defined electromagnetic measurements with a numerical model of the device. The inverse problem minimizes iteratively the difference between the measurements and the simulated quantities, using a minimization algorithm, see section 5 .

For the ideal case, i.e. measurements are noise-free and the mathematical model perfectly represents the reality, the actual magnetic properties of the material may be precisely retrieved. However, neither measurements nor modeling are perfect. The measurements contain noise, and the mathematical model simplifies the reality, i.e. some of the physical phenomena are not correctly modeled. Moreover, some of the model parameters, e.g. related to the geometry, are not known exactly. Their values are uncertain. Consequently, recovering the actual magnetic material properties is not guaranteed.

In the presented inverse problem approach, we propose the use of the Cramér-Rao lower bound technique for 'qualitatively' estimating the error in the recovered material properties, due to the measurement noise and uncertainties in the model parameters, see section 6 . Based on the results obtained using this method, we are able to design a priori the inverse problem. For example, selecting the optimal condition for performing the measurements, and choosing the best measurement modalities which lead to the best recovery results.

Furthermore, we propose two efficient numerical techniques for reducing 'quantitatively' the modeling error in the inverse problem solution. In section 7.1, a deterministic technique is proposed for reducing the modeling error caused by the uncertain model parameter values, by modifying the minimization scheme of the inverse problem. On the other hand, in section 7.2, the stochastic Bayesian approach is utilized for reducing the modeling error originated from the modeling simplifications.

\section{General overview of the forward and inverse problem}

Generally, the problem of computing the model response of a physical system is called a forward problem. On the other hand, the inverse problem theory concerns the problem of making inferences about a physical system starting from 'indirect' noisy measurements (Scales \& Snieder, 2000).

In electromagnetism, the accurate modeling of the electromagnetic phenomena is carried out by solving Maxwell's equations for certain given geometry, sources, and material characteristics. These so-called forward models can be used for analyzing the behavior of the system.

\footnotetext{
${ }^{3}$ The relative errors in the local $H$-measurements are higher than the relative errors in local $B$-measurements, especially for highly non-uniform magnetic field patterns. In practice, the performing accurate local $H$-measurements is not an easy task.
} 


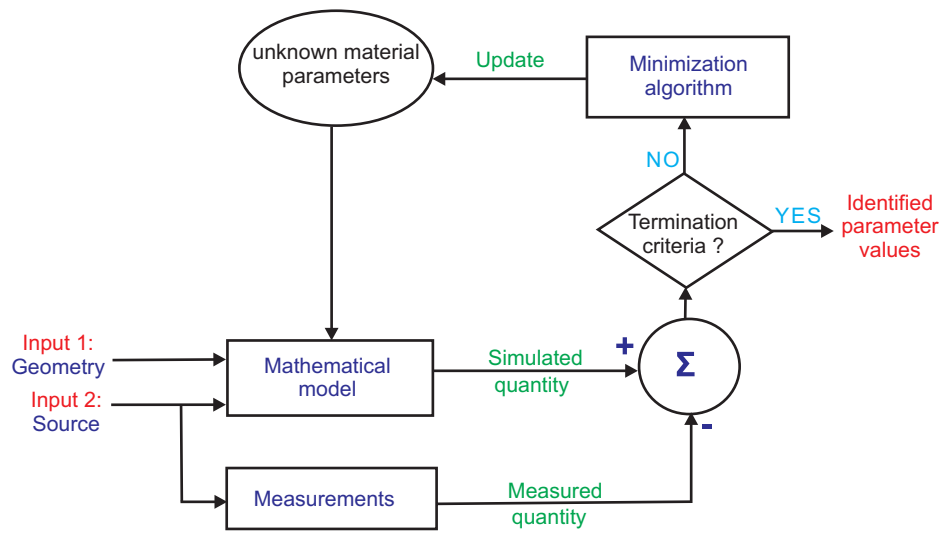

Fig. 2. The detailed schematic diagram of the 'ideal' inverse problem procedure. The termination criteria can be a predefined tolerance on the difference between the measured and simulated quantity.

Moreover, the forward problems also can be used for solving inverse problems. In fact, solving numerically inverse problems has been a subject of research for many years and arises when dealing with more complex systems. Notice that an inverse algorithm is using a certain number of forward model evaluations. The solution of the inverse problem is obtained by proposing an iterative scheme for the identification of the values of the unknown variables (geometrical, sources, or material characteristics) which need to be recovered.

In order to identify a certain unknown parameter of an electromagnetic device, as in an inverse problem, a predefined objective 'cost' function needs to be minimized. This objective function is the quadratic difference between the measured and simulated quantities. ${ }^{4}$

Due to the fact that the aim of this chapter is to characterize the magnetic material properties of an electromagnetic device on its complex geometry, we will restrict ourselves to this aim, i.e. the identification of the geometrical parameters or sources are not studied in this work. These issues have been discussed in (Crevecoeur, 2009), (Yitembe et al., 2011). The detailed schematic diagram of the 'ideal ${ }^{5 \prime}$ inverse problem ${ }^{6}$ for magnetic material characterization, used in this chapter, is shown in Fig. 2.

\footnotetext{
${ }^{4}$ The well known least square non-linear algorithm, Levenberg-Marquardt method with line search (Marquardt, 1963), is often used for minimizing the cost function.

${ }^{5}$ Ideal inverse problem means that the mathematical model of the device represents perfectly the reality, i.e. no modeling error due to the simplification of reality and the model parameters are exactly known. Also, the measurements are assumed to be noise-free. For more detail concerning this issue, see sections 6 and 7.

${ }^{6}$ It is worth mentioning that electromagnetic optimization problems differ from electromagnetic inverse problems. The objective function in optimization problems is based on the minimization or maximization of the target, e.g. efficiency, maximum torque, power per unit volume of an electromagnetic device, coupled through simulated quantities in order to obtain the optimal values for the predefined parameters. The electromagnetic optimization problems are used for designing the electromagnetic devices. However, electromagnetic inverse problems are used for recovering properties of existing electromagnetic devices. In this chapter, we concern only with electromagnetic inverse problems.
} 


\section{Inverse problem formulation}

The behavior of a magnetic system can be represented by a mathematical model with a set of partial differential equations. This model is parameterized by the following model parameters: the unknown parameters $\mathbf{u} \in \mathbb{R}^{p}$, the uncertain parameters $\mathbf{b} \in \mathbb{R}^{q}$, e.g. geometrical model parameters, and the precisely known parameters $\mathbf{d} \in \mathbb{R}^{x}$.

For solving inverse problems, the experimental observations $\mathbf{W} \in \mathbb{R}^{K}$ and the corresponding predicted model responses $\boldsymbol{\Phi} \in \mathbb{R}^{K}$ need to be obtained for the same conditions, e.g. same observation positions, same excitation source, etc. The experimental observations $\mathbf{W}$ of the state of a system are obtained for an excitation source $I_{k}$, and it can be represented by:

$$
W_{k}=\left\{w\left(I_{k}\right)\right\} \quad k=1,2, \ldots, K
$$

with $K$ being the total number of discrete experimental observations.

On the other hand, the predicted response $\boldsymbol{\Phi}$ of a system based on the mathematical model, which involves the solution of the field equations, using the same excitation source $I_{k}$ and the model parameters, can be represented by:

$$
\Phi_{k}(\mathbf{u})=\left\{\phi\left(\mathbf{u}, I_{k}\right)\right\} \quad k=1,2, \ldots, K
$$

In order to estimate the unknown parameters $\mathbf{u}$, an inverse problem has to be solved by iteratively minimizing the quadratic residuals between the experimental observations of the magnetic system $\mathbf{W}$ and the modeled ones $\boldsymbol{\Phi}$. In other words, the functional $\Gamma(\mathbf{u})$

$$
\Gamma(\mathbf{u})=\sum_{k=1}^{K}\left\|W_{k}-\Phi_{k}(\mathbf{u})\right\|^{2}
$$

needs to be minimized:

$$
\widetilde{\mathbf{u}}=\arg \min _{\mathbf{u}} \Gamma(\mathbf{u})
$$

with $\widetilde{\mathbf{u}}$ being the recovered unknown parameters using the inverse approach. The resolution of the inverse procedure highly depends on both measurements and modeling accuracy. $\Gamma$ can depend on the definition of the inverse problem, i.e. the place where the measurements are conducted, the objective function to be minimized, etc. This resolution is due to measurement noise, available uncertainties in the forward mathematical model parameter values, or the simplifications in the used model.

\section{Sources of errors}

In practice, the actual measurements $\mathbf{W}$, can be expressed as:

$$
\mathbf{W}=\boldsymbol{\Phi}\left(\mathbf{u}^{*}\right)+\mathbf{e}
$$

with $\mathbf{e} \in \mathbb{R}^{K}$ being the error vector. The difference e between the simulated signals $\boldsymbol{\Phi}\left(\mathbf{u}^{*}\right)$ with the actual values for the model parameters $\mathbf{u}^{*}$ and the measured signals $\mathbf{W}$ may have two contributions:

$$
\mathbf{e}=\mathbf{e}_{n}+\mathbf{e}_{m}
$$

with $\mathbf{e}_{n}$ being the error due to measurement noise and $\mathbf{e}_{m}$ due to modeling uncertainties. In fact, the modeling error $\mathbf{e}_{m}$ can be divided into two main parts: the modeling error due 
to the uncertainty of some of the model parameters $\mathbf{b}$, and the modeling error due to the simplification of the used mathematical model. Due to $\mathbf{e}$, when minimizing the cost function (Equation 7), the values of the recovered parameters $\widetilde{\mathbf{u}}$ and the actual parameter values $\mathbf{u}^{*}$ are not necessary equal, i.e. $\widetilde{\mathbf{u}} \neq \mathbf{u}^{*}$.

\section{Error estimation in the inverse problem solutions}

As mentioned in section 5, a coupled experimental-numerical inverse approach is formulated in order to characterize the magnetic material of an electromagnetic device. The input of this inverse approach is a set of electromagnetic measurements, while the output is the magnetic material characteristic. It has been observed by numerical experiments "after solving the inverse problem" that the accuracy of the recovered material characteristics appreciably depends on the input nature of the inverse problem, e.g. the objective function to be minimized, the positions where the local magnetic measurements are carried out, etc, (Abdallh et al., 2009), (Abdallh et al., 2010d). However, it is desirable to define and estimate this accuracy a priori "before solving the inverse problem".

Generally, the inverse problem is an ill-posed problem, i.e. small deviation in the input data (measured quantities) leads to a considerable deviation in the output data (recovered material parameters). Consequently, the input of the inverse problem, i.e. 'measurements', and the mathematical model have to be perfectly accurate. However, neither measurements nor mathematical models are strictly accurate: measurements are distorted by measurement noise, and responses in the electromagnetic device model exhibit variations due to uncertainties of some parameter values used in these model calculations. Also, the model of an electromagnetic device does not simulate perfectly all phenomena as in the reality.

In this section, we aim at estimating a priori the error of the recovered magnetic material properties due to the measurement noise and the uncertainties in the electromagnetic device model parameter values. ${ }^{7}$ State-of-the-art of the Monte Carlo simulations are able to achieve this goal but computations may become prohibitive, especially when dealing with time demanding numerical forward electromagnetic models, e.g. finite element models. Therefore, a mathematical technique based on the stochastic Cramér-Rao bound (sCRB) is presented for estimating the uncertainty in the inverse problem solutions. This SCRB offers the lower bound of the error within a rather small computational time compared to the well-know time-demanding techniques such as Monte Carlo simulations applied in e.g. (Leach et al., 2009), stochastic finite element method (Beck \& Woodbury, 1998), polynomial chaos decomposition (Gaignaire et al., 2010).

In the stochastic Cramér-Rao bound method, it is assumed that the identification procedure is affected by the uncertainties in the measurements, and uncertainties in the model parameters. Several types of measurement uncertainties can be considered, in particular systematic and random uncertainty.

A systematic measurement uncertainty can be defined as a reproducible error that biases the measured value in a given direction (NIST, 2000), i.e. a systematic overestimation or underestimation of the true value. A systematic measurement uncertainty is by definition reproducible. Therefore, it cannot be reduced by averaging the values of a large number of

\footnotetext{
${ }^{7}$ In this section, we assume that the model is perfect, i.e. all physical phenomena are included in the model. The inaccuracy in the model structure is discussed in section 7.2.
} 
measurements. However, the reproducible nature of the systematic measurement uncertainty makes it possible to estimate the bias on the measured value by means of a calibration procedure (Lauwagie, 2003). The results presented in this chapter restrict to cases where the measurement uncertainties contain only the random component 'noise'.

When carrying out magnetic measurements, noise can be caused by vibrations of steel sheets, noise originating from excitation current, noise due to the stray field, air flux noise, environmental noise, etc. (Fiorillo, 2004). Here, the measurement noise is assumed uncorrelated and Gaussian white distributed with zero mean and variance of $\sigma_{n}^{2}$.

Again, the least squares approach (Equation 7) and (Equation 8) may not yield to accurate identification results, because no information about $\mathbf{e}_{n}$ is utilized. So, when minimizing the cost function (Equation 7), the values of the recovered parameters $\widetilde{\mathbf{u}}$ and the actual parameter values $\mathbf{u}^{*}$ are not necessary equal, i.e. $\widetilde{\mathbf{u}} \neq \mathbf{u}^{*}$. Due to the fact of the poor posedness of the inverse problems, the search for the 'accurate' minimum value of $\Gamma(\mathbf{u})$ is difficult, and needs advanced mathematical techniques as regularization tools for determining the minimum and criteria for terminating the search (Engl et al., 1996), (Saitoh, 2007).

Due to this fact, it seems reasonable, when solving inverse problems, to use a criterion which reflects the statistical information available on the measurement noise. Therefore, the use of the Cramér-Rao bound method (CRB) is proposed for quantifying the possible uncertainties on the identified unknown parameter values $\mathbf{u}$. CRB is widely-used in many engineering applications; heat transfer applications (Fadale et al., 1995a), biomedical engineering applications (Radich \& Buckley, 1995), and signal analysis applications (Stoica \& Nehorai, 1989).

\subsection{Traditional Cramér-Rao bound method (CRB)}

In the traditional CRB method, it is assumed that the mathematical modeling is accurate, i.e. $\mathbf{e}_{m}=0$. Hence, (Equation 9) can be rewritten as:

$$
\mathbf{W}=\boldsymbol{\Phi}\left(\mathbf{u}^{*}\right)+\mathbf{e}_{n}
$$

It is thus possible to represent the forward model as $\boldsymbol{\Phi}(\mathbf{u})+\mathbf{e}_{n}$ with the incorporation of the measurement noise. Since the measurement noise is supposed to be known, the parameter vector, to be estimated, of this model is still $\mathbf{u}$.

We denote the unbiased estimation of this parameter vector 'after solving the inverse problem' by $\widetilde{\mathbf{u}}$. The Cramér-Rao inequality theorem states that the covariance matrix of the deviation between the true and the estimated parameters is bounded from below by the inverse of the Fisher information matrix F (Goodwin \& Payne, 1977), (Strang, 1986):

$$
E\left\{\left(\widetilde{\mathbf{u}}-\mathbf{u}^{*}\right)\left(\widetilde{\mathbf{u}}-\mathbf{u}^{*}\right)^{T}\right\} \geq \mathbf{F}^{-1}
$$

where $E$ is the expectation. The Fisher information matrix $\mathbf{F}$ gives an indication about the probability of observing noisy measurements $\mathbf{W}$ given the model $\boldsymbol{\Phi}$ and the unknown model parameters $\mathbf{u}$. The probability distribution function of the random variable $\mathbf{W}$ given the value of $\mathbf{u}$, which is also the likelihood function of $\mathbf{u}$, is a function $L(\mathbf{W} \mid \mathbf{u})$. Indeed, the best estimates of the unknown parameter values $\mathbf{u}$ are those that maximizes the probability density function of the measurements $L(\mathbf{W} \mid \mathbf{u})$. This estimate of $\mathbf{u}$ makes the measurements most likely and therefore the probability density function $L(\mathbf{W} \mid \mathbf{u})$ is called the likelihood function. 
The Fisher information matrix $\mathbf{F}$ is calculated based on the partial derivative of the log of the likelihood function with respect to the parameter vector $\mathbf{u}$, which can be calculated by (Goodwin \& Payne, 1977):

$$
\mathbf{F}=E\left\{\left[\frac{\partial}{\partial \mathbf{u}} \ln L(\mathbf{W} \mid \mathbf{u})\right]\left[\frac{\partial}{\partial \mathbf{u}} \ln L(\mathbf{W} \mid \mathbf{u})\right]^{T}\right\}
$$

with $\ln L(\mathbf{W} \mid \mathbf{u})$ being the log-likelihood of $\mathbf{W}$ given the parameter vector $\mathbf{u}$. $\quad \mathbf{F}$ is a matrix with $p \times p$ dimensions. The likelihood of the data is normally distributed and is given by $L\left(W_{1}, \ldots, W_{k}\right)$ :

$$
L(\mathbf{W} \mid \mathbf{u})=\prod_{k=1}^{K} \frac{1}{(2 \pi)^{1 / 2} \sigma_{n}} \cdot \exp \left(-\frac{1}{2 \sigma_{n}^{2}}\left[W_{k}-\Phi_{k}(\mathbf{u})\right]^{2}\right)
$$

and can be rewritten as:

$$
L(\mathbf{W} \mid \mathbf{u})=\frac{1}{(2 \pi)^{K / 2}\left(\sigma_{n}\right)^{K}} \cdot \exp \left(-\frac{1}{2 \sigma_{n}^{2}} \sum_{k=1}^{K}\left[W_{k}-\Phi_{k}(\mathbf{u})\right]^{2}\right)
$$

So that the log-likelihood function becomes:

$$
\ln L(\mathbf{W} \mid \mathbf{u})=\text { const }-K \ln \left(\sigma_{n}\right)-\frac{1}{2 \sigma_{n}^{2}} \sum_{k=1}^{K}\left[W_{k}-\Phi_{k}(\mathbf{u})\right]^{2}
$$

So the derivative to $\mathbf{u}$ becomes:

$$
\frac{\partial \ln L(\mathbf{W} \mid \mathbf{u})}{\partial \mathbf{u}}=\frac{1}{\sigma_{n}^{2}} \sum_{k=1}^{K}\left(W_{k}-\Phi_{k}(\mathbf{u})\right) \frac{\partial \Phi_{k}(\mathbf{u})}{\partial \mathbf{u}}
$$

Substituting (Equation 17) in (Equation 13), the Fisher information matrix can be written as (Goodwin et al., 1974):

$$
\begin{aligned}
(F)_{l m}=\sum_{k=1}^{K} & {\left[\left\{\frac{\partial \Phi_{k}}{\partial u_{m}}\right\}^{T} S_{k}^{-1}\left\{\frac{\partial \Phi_{k}}{\partial u_{l}}\right\}\right.} \\
+ & \left.\frac{1}{2} \operatorname{Tr}\left[S_{k}^{-1}\left\{\frac{\partial S_{k}}{\partial u_{l}}\right\} S_{k}^{-1}\left\{\frac{\partial S_{k}}{\partial u_{m}}\right\}\right]\right] \quad l, m=1, \ldots, p
\end{aligned}
$$

since $\sigma_{n}^{2}=E\left\{(1 / K) \sum_{k=1}^{K}\left[W_{k}-\Phi_{k}(\mathbf{u})\right]^{2}\right\}$, with $S_{k}=\sigma_{n}^{2}$ being the measurement variance, $k=$ $1, \ldots, K$.

Usually, the form of the Fisher information matrix $(\mathbf{F})$ is simplified by assuming that the measurement noise is the same for all experimental observations $K$, uncorrelated, and its variance is independent on the unknown parameters $\mathbf{u}$, i.e. $S_{k}=\sigma_{n}^{2}$ and $\frac{\partial S_{k}}{\partial u_{p}}=0$ (Alifanov et al., 1995), (Fadale et al., 1995b). Thus, under these conditions, the Fisher information matrix (F) reduces to the classical form:

$$
(F)_{l m} \cong \sum_{k=1}^{K}\left[\left\{\frac{\partial \Phi_{k}}{\partial u_{m}}\right\}^{T} S_{k}^{-1}\left\{\frac{\partial \Phi_{k}}{\partial u_{l}}\right\}\right] \quad l, m=1, \ldots, p
$$


Using the inequality of Cramér-Rao bound (Equation 12), the lower bound for the $p$ variances of the unknown parameters $\sigma_{\mathrm{u}, \mathrm{F}}^{2}$ is given by (Stoica \& Nehorai, 1989):

$$
\sigma_{\mathrm{u}, \mathrm{F}_{i i}}^{2} \geq\left(\mathbf{F}^{-1}\right)_{i i} \quad i=1, \ldots, p
$$

$\mathbf{F}^{-1}$ expresses the lower bound for the covariance matrix of the unknown parameters where the variances of each unknown parameter can be deduced as the diagonal elements of $\mathbf{F}^{-1}$.

\subsection{Stochastic Cramér-Rao bound method (sCRB)}

Besides the recovery errors due to measurement uncertainties elaborated in section 6.1, errors are also introduced by the modeling uncertainty.

Specifically, the accuracy of the modeled response depends upon the numerical algorithm and the degree of approximation used, e.g. finite difference or finite element, coarse or fine discretization, etc. These errors can be reduced by using very fine discretizations, inclusion of more accurate material models, etc. This issue is discussed in more detail in section 7.2. In addition to these errors, the modeled response also exhibits variations which are due to the uncertainties in the uncertain parameters $\mathbf{b}$ used in these model calculations. We assume in this chapter that the mathematical forward model algorithm is exact by using a very fine mathematical mode so that the estimated values of the parameters are only influenced by the uncertain model parameters.

The traditional CRB method can be extended when dealing with stochastic uncertain model parameters b, see (Fadale et al., 1995a), (Emery et al., 2000) with an unbiased estimator $\mathbf{u}$. The forward problem becomes now $\boldsymbol{\Phi}(\mathbf{u}, \mathbf{b})+\mathbf{e}_{n}$. The predefined mean value of the uncertain parameters $\mathbf{b}$ defines the model $\boldsymbol{\Phi}$. We assume that the random $\mathbf{b}$ is a Gaussian prior with mean and variance values of $\mu_{b}$ and $\sigma_{b}^{2}$, respectively. In this case, the forward problem becomes $\boldsymbol{\Phi}\left(\mathbf{u}, \mu_{b}\right)+\mathbf{e}_{n}+\mathbf{e}_{m}$. Since $\mathbf{e}_{n}$ and $\mathbf{e}_{m}$ are normally distributed, it is possible to express the total uncertainty $\mathbf{e}_{t}$ in a normal distribution function as well, i.e. $\mathbf{e}_{t}=\mathbf{e}_{n}+\mathbf{e}_{m}$, with $\mathbf{e}_{t} \sim \mathcal{N}\left(\mu_{t}, \sigma_{t}\right)$, where $\mathcal{N}$ is the normal distribution function.

Similar to the derivation of the traditional CRB presented in previous section, the extended Fisher information matrix can be obtained. Here, the extended Fisher information matrix $\mathbf{M}$ is calculated based on the partial derivative of the log of the likelihood function with respect to the parameter vector $\mathbf{u}$, which can be calculated by (Goodwin \& Payne, 1977):

$$
\mathbf{M}=E\left\{\left[\frac{\partial}{\partial \mathbf{u}} \ln L(\mathbf{W} \mid \mathbf{u})\right]\left[\frac{\partial}{\partial \mathbf{u}} \ln L(\mathbf{W} \mid \mathbf{u})\right]^{T}\right\}
$$

with $\ln L(\mathbf{W} \mid \mathbf{u})$ being the $\log$-likelihood of the $\mathbf{W}$ given the parameter vector $\mathbf{u}$. $\mathbf{M}$ is a matrix with $p \times p$ dimensions. The likelihood of the data is normally distributed and is given by $L\left(W_{1}, \ldots, W_{k}\right)$ :

$$
L(\mathbf{W} \mid \mathbf{u})=\prod_{k=1}^{K} \frac{1}{(2 \pi)^{1 / 2} \sigma_{t}} \cdot \exp \left(-\frac{1}{2 \sigma_{t}^{2}}\left[W_{k}-\Phi_{k}\left(\mathbf{u}, \mu_{b}\right)\right]^{2}\right)
$$

and can be rewritten as:

$$
L(\mathbf{W} \mid \mathbf{u})=\frac{1}{(2 \pi)^{K / 2}\left(\sigma_{t}\right)^{K}} \cdot \exp \left(-\frac{1}{2 \sigma_{t}^{2}} \sum_{k=1}^{K}\left[W_{k}-\Phi_{k}\left(\mathbf{u}, \mu_{b}\right)\right]^{2}\right)
$$


So that the log-likelihood function becomes:

$$
\ln L(\mathbf{W} \mid \mathbf{u})=\text { const }-K \ln \left(\sigma_{t}\right)-\frac{1}{2 \sigma_{t}^{2}} \sum_{k=1}^{K}\left[W_{k}-\Phi_{k}\left(\mathbf{u}, \mu_{b}\right)\right]^{2}
$$

So the derivative to $\mathbf{u}$ becomes:

$$
\frac{\partial \ln L(\mathbf{W} \mid \mathbf{u})}{\partial \mathbf{u}}=\frac{1}{\sigma_{t}^{2}} \sum_{k=1}^{K}\left(W_{k}-\Phi_{k}\left(\mathbf{u}, \mu_{b}\right)\right)\left(\frac{\partial \Phi_{k}\left(\mathbf{u}, \mu_{b}\right)}{\partial \mathbf{u}}\right)
$$

Substituting (Equation 25) in (Equation 21), the extended Fisher information matrix can be written as (Emery et al., 2000):

$$
\begin{aligned}
(M)_{l m}=\sum_{k=1}^{K} & {\left[\left\{\frac{\partial \Phi_{k}}{\partial u_{m}}\right\}^{T} V_{k}^{-1}\left\{\frac{\partial \Phi_{k}}{\partial u_{l}}\right\}\right.} \\
& \left.+\frac{1}{2} \operatorname{Tr}\left[V_{k}^{-1}\left\{\frac{\partial V_{k}}{\partial u_{l}}\right\} V_{k}^{-1}\left\{\frac{\partial V_{k}}{\partial u_{m}}\right\}\right]\right] \quad l, m=1, \ldots, p
\end{aligned}
$$

since $\sigma_{t}^{2}=E\left\{(1 / K) \sum_{k=1}^{K}\left[W_{k}-\Phi_{k}\left(\mathbf{u}, \mu_{b}\right)\right]^{2}\right\}$, with $\mathbf{V}$ being the covariance matrix of the total uncertainty, and is given by:

$$
\begin{aligned}
V_{k} & =E\left[\left\{e_{t, k}-E\left[e_{t, k}\right]\right\}\left\{e_{t, k}-E\left[e_{t, k}\right]\right\}^{T}\right] \\
& =E\left[\delta \phi_{k} \delta \phi_{k}^{T}\right]+E\left[\delta W_{k} \delta W_{k}^{T}\right]
\end{aligned}
$$

The first-order estimate of the covariance matrix $E\left[\delta \Phi_{k} \delta \Phi_{k}^{T}\right]$ of the predictions is given by:

$$
E\left[\delta \Phi_{k} \delta \Phi_{k}^{T}\right]=\Theta_{k} G \Theta_{k}^{T} \quad k=1,2, \ldots, K
$$

where $\Theta_{k}$ is the sensitivity matrix of the system prediction $\Phi$ with respect to the uncertain parameter $\mathbf{b}$, which can be calculated using any numerical differentiation techniques, e.g. finite difference technique. $\Theta_{k}$ is defined as:

$$
\Theta_{k, q}=\partial \Phi_{k} / \partial b_{q}
$$

while the covariance matrix $E\left[\delta W_{k} \delta W_{k}^{T}\right]$ is equal to the covariance of the measurement noise, i.e. $E\left[\delta W_{k} \delta W_{k}^{T}\right]=S_{k}$. Therefore (Equation 27) can be expressed as:

$$
\begin{gathered}
V_{k}=\Theta_{k} G \Theta_{k}^{T}+S_{k} \\
\frac{\partial V_{k}}{\partial u_{l}}=2 \Theta_{k} G\left(\frac{\partial \Theta_{k}}{\partial u_{l}}\right)^{T}+\frac{\partial S_{k}}{\partial u_{l}} \quad l=1, \ldots, p
\end{gathered}
$$

According to (Emery et al., 2000), the effect of the trace term is very small, and can thus be neglected. The extended Fisher information matrix, M, can then be approximated by:

$$
(M)_{l m} \cong \sum_{k=1}^{K}\left[\left\{\frac{\partial \Phi_{k}}{\partial u_{m}}\right\}^{T} V_{k}^{-1}\left\{\frac{\partial \Phi_{k}}{\partial u_{l}}\right\}\right] \quad l, m=1, \ldots, p
$$


In this situation, a comparison of (Equation 19) and (Equation 32) shows that $V_{k}$ can be considered as the equivalent noise of the experiment.

So, the lower bound for the $p$ variances of the unknown parameter $\sigma_{\mathrm{u}, \mathrm{M}}^{2}$ is (Radich \& Buckley, 1995):

$$
\sigma_{\mathrm{u}, \mathrm{M}_{i i}}^{2} \geq\left(\mathbf{M}^{-1}\right)_{i i} \quad i=1, \ldots, p
$$

In other words, $\mathbf{M}^{-1}$ expresses the lower bound for the covariance matrix of the unknown parameters where the variances of each unknown parameter can be deduced as the diagonal elements of $\mathbf{M}^{-1}$.

\section{Error reduction in the inverse problem solutions}

The material properties characterizing the magnetic circuit of an electromagnetic device can be identified by solving an inverse problem, where sets of measurements are properly interpreted using a forward numerical model of the device, as explained in section 5 .

In practice, two major aspects can reduce the accuracy of the recovered solution when solving the inverse problem, specifically: measurement noise and inaccurate modeling. Measurement noise can be eliminated or reduced to some extend by accurately performing the measurements, see (Abdallh \& Dupré, 2010c). On the other hand, modeling errors are basically originating from two main sources: the uncertain model parameter values, and the way of modeling of the physical phenomena of the electromagnetic device.

In section 6, a stochastic methodology for estimating a priori the uncertainty in the inverse problem solution is presented. Using this stochastic technique, one may be able to reduce the error in the solution of the inverse problem by incorporating, in the real inverse problem, the most accurate measurement modality, or performing the local measurements at the optimal positions. However, this is a qualitative error reduction. In order to reduce the error in the inverse problem solution quantitatively, we propose two approaches in this section.

First, we propose an effective technique, section 7.1, in which the influences of the uncertainties in the geometrical model parameters are minimized. In this proposed approach, the objective function, that needs to be minimized, is adapted 'iteratively' with respect to the uncertain geometrical model parameters.

On the other side, the second approach aims at reducing the modeling error due to the inaccurate modeling, i.e. not all physical phenomena are modeled, or in other words, the model does not exactly simulate the reality. The second approach uses the stochastic Bayesian technique, section 7.2.

\subsection{Minimum path of the uncertainty method (MPU)}

For the reconstruction of the magnetic material characteristics, the sources and the geometry of this electromagnetic device have to be exactly known. However, in practice, the data of the dimensions of the electromagnetic device, provided by the manufacture, may be uncertain. So, one may introduce errors in the modeling due to the geometrical inaccuracy. Consequently, the inverse results may be inaccurate, to some extend, see (Abdallh et al., 2011b). 
Traditionally, the inverse problem is formulated by minimizing iteratively the residual between the measurements and simulated quantities. However, the uncertainty in the geometrical parameters in the forward model alters the shape of the "traditional" objective function and has thus an effect on the values of the recovered magnetic material properties. Therefore, a new formulation of the objective function is needed, in which the impact of the uncertain geometrical model parameters is reduced.

In case of noise-free measurements and correct modeling of the forward problem, the experimental observations of the magnetic system $\mathbf{W}$ can be expressed as

$$
\mathbf{W}=\boldsymbol{\Phi}\left(\mathbf{u}^{*}, \mathbf{b}^{*}, \mathbf{d}\right)
$$

where $\mathbf{u}^{*}$ and $\mathbf{b}^{*}$ are the actual values of the unknown and uncertain model parameters, respectively, while $\mathbf{d}$ are the precisely known model parameters.

Generally, in order to estimate the unknown parameters $\mathbf{u}$, an inverse problem has to be solved by iteratively minimizing the quadratic residuals between the experimental and modeled observations. In other words, the functional

$$
\Gamma_{\text {Trad }}(\mathbf{u})=\|\Phi(\mathbf{u}, \mathbf{b}, \mathbf{d})-\mathbf{W}\|^{2}
$$

needs to be minimized:

$$
\widetilde{\mathbf{u}}=\arg \min _{\mathbf{u}} \Gamma_{\text {Trad }}(\mathbf{u})
$$

with $\widetilde{\mathbf{u}}$ being the recovered values of the unknown model parameters. Particularly, when $\mathbf{b}=\mathbf{b}^{*}$ is satisfied, then the inverse problem is capable of recovering, in noise-free case and with a perfect forward model, the actual unknown parameters, i.e. $\widetilde{\mathbf{u}} \equiv \mathbf{u}^{*}$.

However, in practice, the knowledge of $\mathbf{b}$ is uncertain and the used value can differ from the actual value. For simplicity, assume only one uncertain model parameter, i.e. $q=1$. If we consider an assumed value $b^{\bullet}$, i.e. $b^{\bullet} \neq b^{*}$, then

$$
\mathbf{W}=\boldsymbol{\Phi}\left(\mathbf{u}^{*}, b^{\bullet}, \mathbf{d}\right)+\mathbf{e}_{m}
$$

with $\mathbf{e}_{m}$ being the modeling error, which is the error due to the difference between the used $b^{\bullet}$ and the actual $b^{*}$, i.e. $\Delta b=b^{*}-b^{\bullet}$. Consequently, the inverse problem solution results in inaccurate recovered unknown model parameters $\left(\widetilde{\mathbf{u}} \neq \mathbf{u}^{*}\right)$. Note that (Equation 34) can be rewritten as

$$
\mathbf{W}=\mathbf{\Phi}\left(\mathbf{u}^{*}, b^{\bullet}+\Delta b, \mathbf{d}\right)
$$

or its Taylor expansion to the first order:

$$
\mathbf{W} \simeq \boldsymbol{\Phi}\left(\mathbf{u}^{*}, b^{\bullet}, \mathbf{d}\right)+\left.\frac{\partial \Phi}{\partial b}\right|_{\left(b=b^{\bullet}\right)} . \Delta b
$$

where we neglect the higher order terms $\partial^{2} \boldsymbol{\Phi} / \partial^{2} b ; \ldots$ etc. $\partial \boldsymbol{\Phi} / \partial b$ is the sensitivity of the modeled response $\boldsymbol{\Phi}$ with respect to the uncertain model parameter $b$, and is a measure for the "forward propagation" of the uncertainty to the forward solution.

Indeed, up to the first order, (Equation 39) reflects in a better way the actual forward solution. So that the proposed methodology, which is called minimum path of the uncertainty (MPU) 
(Abdallh et al., 2011a), defines the following objective function $\Gamma_{M P U}(\mathbf{u})$ :

$$
\Gamma_{M P U}(\mathbf{u})=\left\|\Phi\left(\mathbf{u}, b^{\bullet}, \mathbf{d}\right)+\left.\frac{\partial \Phi}{\partial b}\right|_{\left(b=b^{\bullet}\right)} . \Delta b-\mathbf{W}\right\|^{2}
$$

The proposed methodology is mainly based on adapting, at each iteration step, the objective function that needs to be minimized with respect to the sensitivity of the model responses to the uncertain model parameter $b$. In (Equation 40 ), since $b^{*}$ and consequently $\Delta b$ are unknown in practice, this can not be implemented unless an approximation can be made for the Taylor coefficient of $\Delta b$. As an approximation of $\Delta b$, (Equation 40) can be rewritten as:

$$
\Gamma_{M P U}(\mathbf{u})=\left\|\Phi\left(\mathbf{u}, b^{\bullet}, \mathbf{d}\right)+\left.\alpha\left(\frac{\partial \Phi}{\partial b}\right)\right|_{\left(b=b^{\bullet}\right)}-\mathbf{W}\right\|^{2}
$$

with $\alpha$ being a constant obtained from a linear fitting.

It is possible to make an approximation of the Taylor coefficient $\alpha$ if many measurement samples are available so as to make a linear fitting between the vector $\left(\mathbf{W}-\Phi\left(\mathbf{u}, b^{\bullet}, \mathbf{d}\right)\right)$ and vector $\left.\frac{\partial \Phi}{\partial b}\right|_{b=b^{\bullet}}$, each vector consists of $K$ points. The linear fitting is done at each iteration using the present value of $\mathbf{u}$ at this specific iteration (Abdallh et al., 2012d).

When minimizing the objective function (Equation 41), a path (parameter values $\mathbf{u}^{(j)}$ for the $j^{\text {th }}$ iteration) is followed that is minimally affected by the uncertainties. Indeed, a linear forward model, i.e. $\Phi\left(\mathbf{u}, b^{\bullet}, \mathbf{d}\right)+\left.\alpha\left(\frac{\partial \Phi}{\partial b}\right)\right|_{b=b^{\bullet}}$, is used with the incorporation of the dependence to the uncertain parameter values and with the calculation of $\alpha$.

In the case of implementing more than one uncertain parameter, (Equation 41) can be rewritten as:

$$
\begin{aligned}
\Gamma_{M P U}(\mathbf{u}) & =\| \Phi\left(\mathbf{u}, \mathbf{b}^{\bullet}, \mathbf{d}\right)+\left.\alpha\left(\frac{\partial \Phi}{\partial b_{1}}\right)\right|_{\left(b_{1}=b_{1}^{\bullet}\right)} \\
& +\left.\beta\left(\frac{\partial \Phi}{\partial b_{2}}\right)\right|_{\left(b_{2}=b_{2}^{\bullet}\right)}+\cdots+\left.\gamma\left(\frac{\partial \Phi}{\partial b_{q}}\right)\right|_{\left(b_{q}=b_{q}^{\bullet}\right)}-\mathbf{W} \|^{2}
\end{aligned}
$$

where $\alpha, \beta, \gamma, \ldots$ are the fitting constants.

\subsection{A Bayesian approach for modeling error reduction}

Generally, there are several techniques to model an electromagnetic device, depending on the required accuracy. Analytical and numerical models are often used for modeling an electromagnetic device. Analytical models are computationally fast but less accurate. Numerical models can be divided into several accuracy levels depending on the degree of freedom and discretization. The two or three dimensional finite element (FE) models (2D-FE or 3D-FE) are commonly used with different mesh discretization levels. In general, numerical models are often more accurate than the analytical ones, but much more computational time consuming; the higher the degree of freedom, the more time consuming. 
In order to solve an inverse problem with the highest solution accuracy, one may incorporate the measurements with the most accurate (fine) model, e.g. 3D numerical FE model, of an electromagnetic device. However, the computational time of this approach may be prohibitive. Alternatively, less accurate (coarse) models, e.g. analytical models, can be used with a demerit of an expected higher recovery error compared to the fine models.

In this section, we propose the use of the Bayesian approximation error approach (Nissinen et al., 2008) for improving the inverse problem solution when a coarse model is utilized. The proposed approach adapts the objective function to be minimized with the a priori misfit between fine and coarse forward models, in which the modeling error is represented in a stochastic way. The Bayesian approximation error approach is relatively fast and easy to implement compared to the two-level techniques, such as space mapping (Bandler et al., 2008), manifold mapping (Echeverría et al., 2006), two-level refined direct method (Crevecoeur et al., 2011a), etc.

Assume that we have two computer models of an electromagnetic device; a fine and a coarse model. The fine model is assumed to be close to the reality where we assume that the modeling error in the fine model is negligible ${ }^{8}$. However, the modeling error in the coarse model is refereed to the misfit between the fine and coarse forward model responses. We present the stochastic modeling error in section 7.2.1.

\subsubsection{Stochastic representation of the modeling error}

The fine and coarse forward model responses, $\boldsymbol{\Phi}_{f} \in \mathbb{R}^{K}$ and $\boldsymbol{\Phi}_{c} \in \mathbb{R}^{K}$ respectively, depend on u. Since the exact value of $\mathbf{u}$ is not known, we assume $Z$ hypothetical values of the unknown model parameters, with $\hat{\mathbf{u}}_{z},(z=1, \ldots, Z)$ being a hypothetical value. These $Z$ hypothetical values are chosen in a such a way that they are random and cover the domain defined by the lower and upper bounds of these parameters.

The error between the fine and coarse forward models, at each model observation $k$ and at each test value $\hat{\mathbf{u}}_{z}$, can be represented by:

$$
e_{m, k}\left(\hat{\mathbf{u}}_{z}\right)=\Phi_{f, k}\left(\hat{\mathbf{u}}_{z}\right)-\Phi_{c, k}\left(\hat{\mathbf{u}}_{z}\right), \quad(k=1, \ldots, K), \quad(z=1, \ldots, Z)
$$

By performing $Z$ coarse and fine forward model computations, and assuming that the modeling error at each model observation $k\left(e_{m, k}\right)$ follows the normal distribution, i.e. $\left(e_{m, k} \sim\right.$ $\left.\mathcal{N}\left(\mu_{m, k}, \sigma_{m, k}^{2}\right)\right)$, one may calculate the mean modeling error and its covariance, $\mu_{m, k}$ and $\sigma_{m, k}^{2}$ respectively.

$$
\begin{gathered}
\mu_{m, k}=\frac{1}{Z} \sum_{z=1}^{Z} e_{m, k}\left(\hat{\mathbf{u}}_{z}\right) \\
\sigma_{m, k}^{2}=\frac{1}{Z} \sum_{z=1}^{Z}\left(e_{m, k}\left(\hat{\mathbf{u}}_{z}\right)-\mu_{m, k}\right)^{2}
\end{gathered}
$$

\footnotetext{
${ }^{8}$ In fact, it is impossible to construct an exact computer model combining the whole physical phenomena as in the reality. However, in order to solve an inverse problem, a computer model is needed with the highest possible accuracy: the most accurate model give rise to the most accurate recovery results.
} 
Based on the calculated $\mu_{m, k}$ and $\sigma_{m, k^{\prime}}^{2}$ the approximate overall probability distribution function (PDF), at each model observation $k$, can be visualized as:

$$
f\left(e_{m, k}\right)=\frac{1}{\sqrt{2 \pi \sigma_{m, k}^{2}}} \cdot \exp ^{\left(-\left(e_{m, k}-\mu_{m, k}\right)^{2} /\left(2 \sigma_{m, k}^{2}\right)\right)}
$$

The assumption of the modeling error to be Gaussian distributed is a possible means of statistically expressing the modeling error, see (Nissinen et al., 2008). The vector representations of the modeling mean error and its covariance at all model observations $K$ are:

$$
\overline{\boldsymbol{\mu}}_{m}=\left[\mu_{m, 1}, \mu_{m, 2}, \ldots, \mu_{m, K}\right]^{T}, \overline{\boldsymbol{\sigma}}_{m}^{2}=\left[\sigma_{m, 1}^{2}, \sigma_{m, 2}^{2}, \ldots, \sigma_{m, K}^{2}\right]^{T}
$$

Since the modeling error is assumed uncorrelated, i.e. $e_{m, i}$ does not depend on $e_{m, j}(i, j=$ $1, \ldots, K, i \neq j)$, the covariance matrix of the modeling error $\left(\overline{\boldsymbol{\Sigma}}_{m}^{2} \in \mathbb{R}^{K \times K}\right)$ can be written as: $\overline{\boldsymbol{\Sigma}}_{m}^{2}=\operatorname{diag}\left(\sigma_{m, 1}^{2}, \sigma_{m, 2}^{2}, \ldots, \sigma_{m, K}^{2}\right)$. The mean and the covariance of the modeling error are used in the following section for the modeling error compensation.

\subsubsection{Bayesian approach: Traditional and approximation error}

As mentioned before, the actual measurements $\mathbf{W}$ can be expressed as:

$$
\mathbf{W}=\boldsymbol{\Phi}\left(\mathbf{u}^{*}\right)+\mathbf{e}
$$

with e being the uncertainty "error" vector. A possible difference between the simulated signals $\boldsymbol{\Phi}\left(\mathbf{u}^{*}\right)$ with the actual model parameters $\mathbf{u}^{*}$, and the measured signals $\mathbf{W}$, can arise from two reasons, and is denoted by:

$$
\mathbf{e}=\mathbf{e}_{n}+\mathbf{e}_{m}
$$

with $\mathbf{e}_{n}$ being the uncertainty due to the measurement noise and $\mathbf{e}_{m}$ being the uncertainty due to modeling uncertainties. Due to the random nature of the measurement noise, it is assumed to be normally white distributed with zero mean $\left(\bar{\mu}_{n, k}=0\right)$ and a covariance of $\bar{\sigma}_{n, k}^{2}$ i.e. $\left(\mathbf{e}_{n, k} \sim \mathcal{N}\left(0, \bar{\sigma}_{n, k}^{2}\right)\right.$ ) (Abdallh et al., 2012c). Similarly,

$$
\bar{\mu}_{n}=\left[\mu_{n, 1}, \mu_{n, 2}, \ldots, \mu_{n, K}\right]^{T}=\mathbf{0}, \bar{\sigma}_{n}^{2}=\left[\sigma_{n, 1}^{2}, \sigma_{n, 2}^{2}, \ldots, \sigma_{n, K}^{2}\right]^{T}
$$

Since the measurement noise is assumed uncorrelated, i.e. $e_{n, i}$ does not depend on $e_{n, j}(i, j=$ $1, \ldots, K, i \neq j)$, the covariance matrix of the measurement noise $\left(\bar{\Sigma}_{n}^{2} \in \mathbb{R}^{K \times K}\right)$ can be written as: $\overline{\boldsymbol{\Sigma}}_{n}^{2}=\operatorname{diag}\left(\sigma_{n, 1}^{2}, \sigma_{n, 2}^{2}, \ldots, \sigma_{n, K}^{2}\right)$.

The modeling error is also represented in normal distribution, see section 7.2.1. Due to these uncertainties, when minimizing the cost function (Equation 35), the values of the recovered parameters $\widetilde{\mathbf{u}}$ and $\mathbf{u}^{*}$ are not necessary equal, i.e. $\widetilde{\mathbf{u}} \neq \mathbf{u}^{*}$. Therefore, we propose the use of the statistical Bayesian approach.

\section{Traditional Bayesian approach:}

In the Bayesian framework, the identification problem is seen as a statistical inference problem, sometimes referred to as stochastic regularization (Emery et al., 2007), in which the measurements and the modeled response are assumed to be random (Nissinen et al., 
2008). In the traditional Bayesian approach, the modeling error is assumed to be negligible, i.e. $\mathbf{W}=\boldsymbol{\Phi}\left(\mathbf{u}^{*}\right)+\mathbf{e}_{n}$.

In the well known Bayes' formula, the posterior probability density function of the measurements $\mathbf{W}$ given the unknown model parameters $\mathbf{u}$ ' $P(\mathbf{u} \mid \mathbf{W})^{\prime}$ ' is given by (Kaipio \& Somersalo, 2005):

$$
P(\mathbf{u} \mid \mathbf{W})=\frac{P(\mathbf{u}) P(\mathbf{W} \mid \mathbf{u})}{P(\mathbf{W})}
$$

which can be written in a non-normalized form:

$$
P(\mathbf{u} \mid \mathbf{W}) \propto P(\mathbf{u}) P(\mathbf{W} \mid \mathbf{u})
$$

with $P(\mathbf{u})$ being the prior probability density function of the unknown model parameters. In our application, no information is given for the $P(\mathbf{u})$. So, we assume that the unknown model parameters follow the uniform distribution between lower and upper bounds:

$$
P(\mathbf{u})=\frac{1}{\mathbf{u}_{U B}-\mathbf{u}_{L B}}, \quad \mathbf{u} \in\left[\mathbf{u}_{L B}, \mathbf{u}_{U B}\right]
$$

with $\mathbf{u}_{L B}$ and $\mathbf{u}_{U B}$ being the lower and upper bounds of the unknown model parameters, respectively, which can be known from the reasonable physical representation of $\mathbf{u}$.

Assuming that the measurement noise $\mathbf{e}_{n}$ does not depend on the unknown model parameters $\mathbf{u}$, the likelihood density function of the measurements $\mathbf{W}$ given the unknown model parameters $\mathbf{u}$ can be written as (Kaipio \& Somersalo, 2005):

$$
\begin{aligned}
P(\mathbf{W} \mid \mathbf{u})= & \frac{1}{(2 \pi)^{K / 2} \prod_{k=1}^{K} \sigma_{n, k}} \\
& \cdot \exp \left(-\frac{1}{2}\left[\left[\mathbf{W}-\boldsymbol{\Phi}(\mathbf{u})-\overline{\boldsymbol{\mu}}_{n}\right]^{T}\left(\overline{\boldsymbol{\Sigma}}_{n}^{2}\right)^{-1}\left[\mathbf{W}-\boldsymbol{\Phi}(\mathbf{u})-\overline{\boldsymbol{\mu}}_{n}\right]\right]\right)
\end{aligned}
$$

Therefore, in order to solve this inverse problem, the maximum a posteriori (MAP) estimates is used, in which the MAP of the unknown model parameters $\mathbf{u}$ is given by:

$$
\mathbf{u}_{\mathrm{MAP}}=\arg \max _{\mathbf{u}} P(\mathbf{u} \mid \mathbf{W})
$$

Substituting (Equation 52) and (Equation 54) in (Equation 55), and $\left(\bar{\mu}_{n}=0\right)$ :

$$
\begin{aligned}
\mathbf{u}_{\mathrm{MAP}, \operatorname{Trad}} & =\arg \max _{\mathbf{u}} P(\mathbf{W} \mid \mathbf{u}) \\
& =\arg \max _{\mathbf{u}}\left\{\exp \left(-\frac{1}{2}\left[[\mathbf{W}-\boldsymbol{\Phi}(\mathbf{u})]^{T}\left(\overline{\boldsymbol{\Sigma}}_{n}^{2}\right)^{-1}[\mathbf{W}-\boldsymbol{\Phi}(\mathbf{u})]\right]\right\}\right. \\
& =\arg \min _{\mathbf{u}}\left\{[\mathbf{W}-\boldsymbol{\Phi}(\mathbf{u})]^{T}\left(\overline{\boldsymbol{\Sigma}}_{n}^{2}\right)^{-1}[\mathbf{W}-\mathbf{\Phi}(\mathbf{u})]\right\} \\
& =\arg \min _{\mathbf{u}}\left\|\mathbf{L}_{n}(\mathbf{W}-\boldsymbol{\Phi}(\mathbf{u}))\right\|^{2}
\end{aligned}
$$

with $\mathbf{L}_{n}$ being the Cholesky factor of the covariance of the measurement noise, i.e. $\left(\bar{\Sigma}_{n}^{2}\right)^{-1}=\mathbf{L}_{n}^{T} \mathbf{L}_{n}$. Solution of (Equation 56) is the recovered model parameter $\left(\mathbf{u}_{\mathrm{MAP}}\right.$ Trad $\left.\equiv \widetilde{\mathbf{u}}\right)$ using the inverse problem in the traditional Bayesian framework. 


\section{Bayesian approximation error approach:}

In the traditional Bayesian approach, the modeling error $\mathbf{e}_{m}$ was assumed to be negligible. However, in the Bayesian approximation error approach, the $\mathbf{e}_{m}$ is taken into account. As discussed earlier in section 7.2.1, the modeling error exists only when the coarse model is incorporated in the inverse problem, so (Equation 48) can be rewritten as:

$$
\mathbf{W}=\boldsymbol{\Phi}_{c}\left(\mathbf{u}^{*}\right)+\mathbf{e}_{n}+\mathbf{e}_{m}
$$

Due to the Gaussian distribution of both the measurement noise and modeling error, the overall error e is therefore also Gaussian distributed. Similarly, (Equation 56) can be reformulated as follows:

$$
\begin{aligned}
\mathbf{u}_{\text {MAP, Compensated }} & =\arg \max _{\mathbf{u}} P(\mathbf{W} \mid \mathbf{u}) \\
& =\arg \min _{\mathbf{u}}\left\|\mathbf{L}_{n+m}\left(\mathbf{W}-\boldsymbol{\Phi}_{c}(\mathbf{u})-\overline{\boldsymbol{\mu}}_{m}\right)\right\|^{2}
\end{aligned}
$$

with $\mathbf{L}_{n+m}$ being the Cholesky factor of the covariance of the overall error, i.e. $\left(\overline{\boldsymbol{\Sigma}}_{n}^{2}+\overline{\boldsymbol{\Sigma}}_{m}^{2}\right)^{-1}=\mathbf{L}_{n+m}^{T} \mathbf{L}_{n+m}$. Solution of (Equation 58) is the recovered model parameter $\left(\mathbf{u}_{\text {MAP, Compensated }} \equiv \widetilde{\mathbf{u}}\right)$ using the inverse problem in the Bayesian approximation error approach, in which the modeling error is compensated.

\section{Magnetic material characteristics}

There are several properties of the material to be recovered, however, we focus only on the magnetic properties. Specifically, the single-valued $B-H$ curve, the hysteresis loops and loss parameters are being recovered here. In order to identify the magnetic characteristics of a material using the inverse problem approach, the magnetic parameters need to be formulated in mathematical formulas to be used in the model.

1. Single-valued $B-H$ curve:

The single-valued nonlinear constitutive characteristic of the $B-H$ curve (normal magnetizing characteristic), to be reconstructed by the inverse problem, is modeled by means of three parameters $\mathbf{u}_{1}=\left[H_{0}, B_{0}, v\right]$ of the (non-full) power-series formula (Abdallh et al., 2010a):

$$
\frac{H}{H_{0}}=\left(\frac{B}{B_{0}}\right)+\left(\frac{B}{B_{0}}\right)^{v}
$$

2. Hysteretic characteristic:

The hysteretic characteristic of the magnetic material, is modeled by means of five parameters $\mathbf{u}_{2}=\left[a, b, c, k_{1}, k_{2}\right]$ of the Lorentzian distribution of the scalar Preisach model (Abdallh et al., 2010d):

$$
P(\alpha, \beta)=\frac{k_{1}}{\left(1+\left(\frac{\alpha-a}{b}\right)^{2}\right)\left(1+\left(\frac{\beta+a}{b}\right)^{2}\right)}+\delta_{\alpha, \beta} \frac{k_{2}}{1+\left(\frac{\alpha}{c}\right)^{2}}
$$

where $P(\alpha, \beta)$ and $\delta_{\alpha, \beta}$ are the Preisach distribution function and the Kronecker delta symbol, respectively (Bertotti, 1998). 


\section{Loss parameters:}

The well-known Bertotti loss model, in its simplified form, is used for loss characterization (Bertotti, 1988):

$$
P_{l p}=a_{1} B_{l p}^{\alpha_{1}} f+b_{1} B_{l p}^{2} f^{2}+c_{1} B_{l p} f\left(\sqrt{\left(1+d_{1} B_{l p} f\right)}-1\right)
$$

with $B_{l p}$ and $f$ being the local peak magnetic induction in $(\mathrm{T})$ and the frequency in $(\mathrm{Hz})$, respectively. $P_{l p}$ is the local iron loss. $\mathbf{u}_{3}=\left[a_{1}, \alpha_{1}, b_{1}, c_{1}, d_{1}\right]$ is the vector of the loss model parameters. The values of these parameters are unknown and need to be identified using an inverse approach.

\section{Application}

The proposed inverse methodology is applied here into the following application, i.e. an EI electromagnetic core inductor, for the complete identification of the magnetic properties of the magnetic material. However, the proposed methodology can be applied for any other application, e.g. a permanent magnet synchronous machine (Sergeant et al., 2009), a switched reluctance motor (Abdallh et al., 2011c), (Abdallh et al., 2011a), an asynchronous motor (Abdallh et al., 2012b).

\subsection{Problem definition}

The magnetic material characteristics (the normal magnetizing curve, the hysteretic characteristics and the loss parameters) of the EI electromagnetic core inductor, shown in Fig. 3 , are unknown and need to be recovered using the inverse approach.

In this application, we consider two magnetic measurements, i.e. the local and global measurements, as being the input of the inverse problem. The local magnetic induction measurements are carried out using the needle probe method as described in (Abdallh \& Dupré, 2010c), at several positions on the EI profile. Global measurements use the current and voltage measurements, i.e. no local measurements are performed.

In practice, the uncertainties of all geometrical model parameters of the EI core are magnetically of second order, except the values of the two air gaps, $g_{1}$ and $g_{2}$. The mean value of the $g_{1}$ is given by the manufacturer, however the mean value of the $g_{2}$ is the thickness of the spacer inserted between the E and I yokes. In this study, we use $g_{2}$ as 0 or $0.25 \mathrm{~mm}$. It is assumed that the local or global measurements are corrupted by Gaussian noise with zero mean and a standard deviation of $\sigma_{n, l o c a l}$ or $\sigma_{n, g l o b a l}$, respectively. Moreover, the standard deviations of the uncertain geometrical model parameters are denoted by $\sigma_{g_{1}}$ and $\sigma_{g_{2}}$.

\subsection{Traditional inverse problem formulation}

The three magnetic material parameters of the single-valued $B-H$ characteristic $\mathbf{u}_{1}=$ $\left[H_{0}, B_{0}, v\right]$, the five parameters of the Lorentzian distribution of the scalar Preisach model $\mathbf{u}_{2}=\left[a, b, c, k_{1}, k_{2}\right]$, and the five loss parameters $\mathbf{u}_{3}=\left[a_{1}, \alpha_{1}, b_{1}, c_{1}, d_{1}\right]$ are recovered using the inverse problem approach. The unknown parameter values can be retrieved by iteratively minimizing predefined objective functions. The objective functions are formulated as the quadratic difference between the measured and the simulated local magnetic induction 


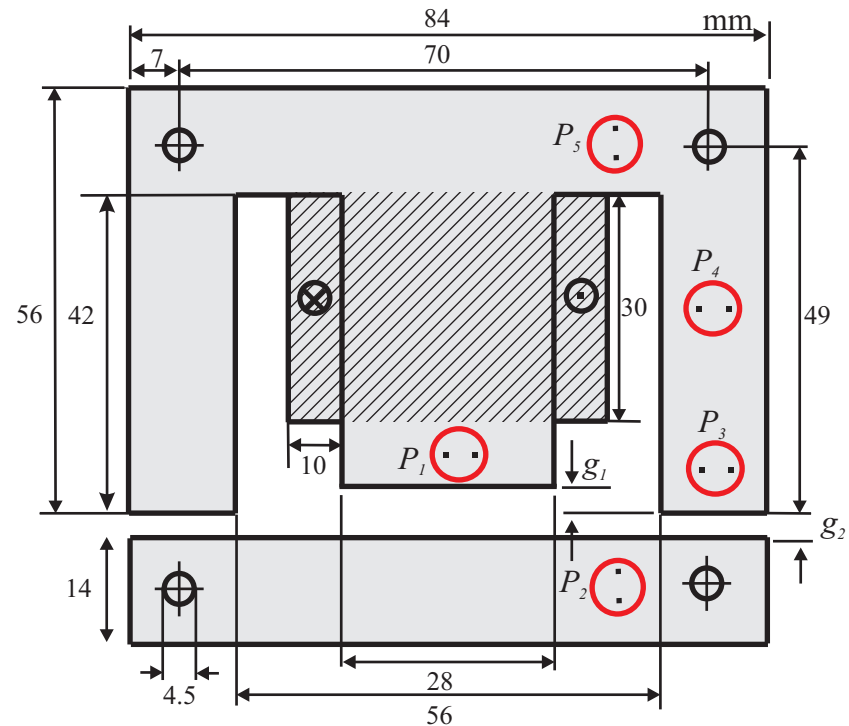

Fig. 3. Schematic diagram of the studied EI electromagnetic core inductor.

at a specific condition.

$$
\widetilde{\mathbf{u}}_{i}=\arg \min _{\mathbf{u}_{i}} \Gamma\left(\mathbf{u}_{i}\right), \quad i=[1,2,3]
$$

where $\widetilde{\mathbf{u}}_{1}, \widetilde{\mathbf{u}}_{2}$ and $\widetilde{\mathbf{u}}_{3}$ are the 'recovered' magnetic material parameters of the single-valued $B-H$ curve, the hysteretic characteristics and loss parameters, respectively.

In the following, we formulate four objective functions. The first and second objective functions are devoted for recovering $\mathbf{u}_{1}$. The third and fourth objective functions are used for recovering $\mathbf{u}_{2}$ and $\mathbf{u}_{3}$, respectively.

1. First objective function:

The first objective function $\Gamma_{1}$ is implemented using the amplitude $\left(i_{k}\right)$ of the $k^{\text {th }}$ sinusoidal excitation current $(k=1, \ldots, K=40)$ and the local magnetic induction measurements, at a fixed position as shown in Fig. 3:

$$
\Gamma_{1}\left(\mathbf{u}_{1}\right)=\sum_{k=1}^{K}\left\|B_{S}\left(i_{k}, \mathbf{u}_{1}\right)-B_{m}\left(i_{k}\right)\right\|_{\text {position } j}^{2} j=[1,2, \ldots, 5]
$$

with $B_{m}\left(i_{k}\right)$ being the measured peak magnetic induction value of the $k^{\text {th }}$ excitation current and $B_{s}\left(i_{k}, \mathbf{u}_{1}\right)$ being the corresponding simulated local flux densities using the numerical model and the material parameter values $\mathbf{u}_{1}$.

2. Second objective function:

The second objective function $\left(\Gamma_{2}\right)$ is implemented using global measurements of the excitation current $I$ and the voltage $V$ of the excitation winding, where no local measurements are used. This global measurement gives rise to the flux density linked with the excitation winding: 


$$
\begin{gathered}
\phi_{m}(t)=\left(1 / N_{1}\right)\left[\int_{0}^{t} V(\tau) d \tau-R \int_{0}^{t} I(\tau) d \tau\right] \\
\Gamma_{2}\left(\mathbf{u}_{1}\right)=\sum_{k=1}^{K}\left\|\phi_{s}\left(i_{k}, \mathbf{u}_{1}\right)-\phi_{m}\left(i_{k}\right)\right\|^{2}
\end{gathered}
$$

with $\phi_{m}\left(i_{k}\right)$ being the measured peak magnetic flux value of the $k^{\text {th }}$ excitation current $(k=$ $1, \ldots, K=40)$, and $\phi_{s}\left(i_{k}, \mathbf{u}\right)$ being the corresponding simulated value. $N_{1}$ and $R$ are the number of turns of the excitation winding $\left(N_{1}=356\right)$, and the resistance of the excitation coil $(R=1.3 \mathrm{Ohm})$, respectively.

3. Third objective function:

The third objective function $\Gamma_{3}$ is constructed as follows:

$$
\Gamma_{3}\left(\mathbf{u}_{2}\right)=\sum_{l=1}^{L} \sum_{n=1}^{T}\left\|b_{s, l}\left(t_{n}, \mathbf{u}_{2}\right)-b_{m, l}\left(t_{n}\right)\right\|_{\text {position } j}^{2} j=[1,2, \ldots, 5]
$$

where $b_{m, l}\left(t_{n}\right)$ is the measured magnetic induction value of the $n^{\text {th }}$ time step $(n=1, \ldots, T=$ 1000) for the $l^{\text {th }}$ magnetization loop and $b_{s, l}\left(t_{n}, \mathbf{u}_{2}\right)$ is the corresponding simulated local flux densities using the numerical model.

4. Fourth objective function:

After recovering $\mathbf{u}_{1}$, the values of $\mathbf{u}_{3}$ are recovered a posteriori. Using a specific value of the frequency $f$ considered in the iron loss measurements, which is not involved in the static computation mode, the iron loss $P_{l p}$, in $\mathrm{W} / \mathrm{kg}$, can be computed using (Equation 61). In order to calculate the overall iron loss of the electromagnetic device $P_{\text {total }}$, in (W), the local iron loss $P_{l, i}$ is multiplied by the mass of the EI inductor $\left(m_{E I}\right)$, i.e. $P_{\text {total }}=P_{l p} \times m_{E I}$. In order to identify the five parameters of the loss model $\mathbf{u}_{3}$, the following objective function is formulated:

$$
\Gamma_{4}\left(\mathbf{u}_{3}\right)=\left\|P_{\text {total }}\left(\mathbf{u}_{3}, \widetilde{\mathbf{u}}_{1}, f\right)-P_{m}(f)\right\|^{2}
$$

with $P_{m}$ being the measured iron loss of the EI core inductor.

\subsection{A priori error estimation}

Depending on the nature of the measurements that are used as input for the inverse problem, a certain resolution or accuracy of the recovered magnetic material parameter values is achieved (Abdallh et al., 2011b). Possible measurements are local (on a specific part of the geometry) and global (whole considered geometry) magnetic measurements. These measurement modalities contain measurement noise which decreases the accuracy of the inverse problem solution. Additionally, the uncertainties of important model parameters, i.e. air gaps, influence the resolution. We assume here $\sigma_{n, l o c a l}=\sigma_{n, \text { global }}=0.025$ and $\sigma_{g_{1}}=\sigma_{g_{2}}=0.025$. Therefore, the sCRB method, presented in section 6.2, is used for selecting $a$ priori the best measurement modality that results in the highest accuracy, taking into account the measurement noise and the geometrical uncertainties.

The optimum measurement modality follows the criterion that this modality has the minimum estimated uncertainty when using the sCRB technique. The prior uncertainty estimation can be implemented before carrying out the real experimental measurements. 
Therefore, numerical experiments are carried out in the sense that the 'numerical measurements' quantities $\left(B_{m}\right.$ or $\left.\phi_{m}\right)$ are 'modeled' as the output of the numerical direct model that has as input the following fictitious single-valued characteristics $\mathbf{u}_{\mathrm{fic}}=$ $[100,1.1,8]$. The output is furthermore corrupted by Gaussian noise with zero mean and a standard deviation of $\sigma_{n}$, assumed as $\sigma_{n, \text { local }}=\sigma_{n, \text { global }}=0.025$. The 'numerical' local magnetic induction measurements are carried out at the five different positions $P_{1}-P_{5}$, shown in Fig. 3.

In fact, and for clarity, the selection process is done in two stages. The first stage aims at determining the optimum placement for carrying out the local measurements among the five different positions $P_{1}-P_{5}$. The second stage aims at selecting the type of the measurement, i.e. global or local measurements. In the second stage, we compare the global measurements with the local measurements at the optimum position.

For the comparative issue, the estimated error $(E E)$ is defined as the percentage estimated error in the recovered material characteristics ${ }^{9}$ and is formulated as:

$$
E E=\left|\frac{R M S_{B H}\left(\mathbf{u}_{\mathrm{fic}}+\sigma_{u, M}\right)}{R M S_{B H}\left(\mathbf{u}_{\mathrm{fic}}\right)}-1\right| \times 100 \%
$$

with $\sigma_{u, M}$ being the lower bound of the standard deviation of the unknown parameters obtained from the sCRB analysis, see (Equation 33). $R M S_{B H}$ is the root mean square of the $B-H$ curve, given by $R M S_{B H}=\sqrt{\sum_{k=1}^{K} \frac{B^{2}\left(H_{k}\right)}{K}}$.

\subsubsection{Optimal needle placement}

Table 1 shows the "theoretical" EE values based on the sCRB due to the measurement noise and the uncertainty in $g_{1}$ and $g_{2}$. It is clear from this table that the local measurements carried out at position $2\left(P_{2}\right)$ results in the best inverse problem results, and the local measurements carried out at positions $1\left(P_{1}\right)$ and $5\left(P_{5}\right)$ result in the worst inverse problem results. Also, Table 1 depicts that the $g_{2}$ is a more critical parameter than $g_{1}$. Similar results are obtained using a deterministic methodology based on first order sensitivity analysis, see (Abdallh et al., 2012a).

\begin{tabular}{|c|c|c|c|c|c|}
\hline Position & 1 & 2 & 3 & 4 & 5 \\
\hline (a) The $E E$ value due to $\sigma_{n}$ and $\sigma_{g_{1}}$ & 44.68 & 34.60 & 39.72 & 36.17 & 36.28 \\
\hline (b) The $E E$ value due to $\sigma_{n}$ and $\sigma_{g_{2}}$ & 50.94 & 43.91 & 51.62 & 44.90 & 44.72 \\
\hline
\end{tabular}

Table 1. The EE values based on the sCRB due to (a) the measurement noise and the uncertainty in $g_{1}\left(\sigma_{n}=0.025, \sigma_{g_{1}}=0.025\right)$, (b) the measurement noise and the uncertainty in $g_{2}\left(\sigma_{n}=0.025, \sigma_{g_{2}}=0.025\right)$.

\subsubsection{Selection measurement modality}

In this section, the sCRB is used for selecting the best measurement modality, i.e. global or local, that results in the best solution. We compare the inverse problem based on global

\footnotetext{
${ }^{9}$ It is worth mentioning that $E E$ can not be calculated in practice, because the knowledge of the sought-after parameters (u) is unknown. However, we use $E E$ in order to estimate the uncertainty in the inverse problem solution in a 'qualitative' way rather than a 'quantitative' way.
} 
measurements, i.e. $\Gamma_{2}$, with the inverse problem based on the local magnetic induction, i.e. $\Gamma_{1}$, at position $2\left(P_{2}\right)$ because at this position the best results are observed. Table 2 shows the "theoretical" $E E$ values based on the sCRB due to the measurement noise and the uncertainty in $g_{2}$. It is clear from this table that the inverse problem based on $\Gamma_{1}$ gives better results than the one based on $\Gamma_{2}$.

\begin{tabular}{|c|c|c|}
\hline Objective function & $\Gamma_{1}$ & $\Gamma_{2}$ \\
\hline The $E E$ value & 43.91 & 53.24 \\
\hline
\end{tabular}

Table 2. The $E E$ values based on the sCRB due to the measurement noise and the uncertainty in $g_{2}\left(\sigma_{n}=0.025, \sigma_{g_{2}}=0.025\right)$ for $\Gamma_{1}$ and $\Gamma_{2}$.

\subsection{Traditional inverse problem}

The magnetic properties of the magnetic material are recovered using the traditional formulation of the inverse approach. Traditional inverse problem means that no quantitative error reduction techniques are used, e.g. MPU or Bayesian techniques. Therefore, the value of $g_{2}$ is kept zero in order to eliminate the error initiates from the uncertainty in its value.

\subsubsection{Identification of the single-valued $B-H$ curve}

In order to validate experimentally the results obtained from the sCRB analysis, three 'traditional' inverse problems are solved. The local magnetic induction measurements at position $1\left(P_{1}\right)$ and position $2\left(P_{2}\right)$ are used for the first two inverse problems. The third inverse problem is solved starting from the global measurements.

Fig. 4 shows the recovered $B-H$ curve, using the measured signals at $P_{1}$ and $P_{2}$ and the global measurements compared to the actual $B-H$ characteristic ${ }^{10}$. It is clear that the recovered characteristic based on measurements at $P_{2}$ is much closer to the actual $B-H$ characteristic than the recovered characteristic based on measurements at $P_{1}$. Moreover, it is also clear that the recovered characteristic based on the local measurements at $P_{2}$ is better than the one based on the global measurements. The values of the recovered parameters compared to the actual values are also shown in Fig. 4. These results validate the sCRB theoretical results.

\subsubsection{Identification of the hysteretic characteristics}

In order to recover the hysteretic characteristics, the 'traditional' inverse problem based on (Equation 62) and (Equation 66) is solved. For simplicity, and the best results, we solve the inverse problem based on the local measurements carried out at position $2\left(P_{2}\right)$. Fig. 5 shows the recovered hysteresis loops of the EI core inductor material using the proposed inverse problem method, compared to the actual magnetic hysteresis loops. A good correspondence between the measured hysteresis loops on the fully wound magnetic ring core, and the recovered one is observed.

\footnotetext{
${ }^{10}$ The actual magnetic characteristics were measured on the fully wound magnetic ring core made from the same material as the material of the EI core inductor, $\mathbf{u}_{1}^{*}=[292.03,1.35,11.99], \mathbf{u}_{2}^{*}=$ $[124.99,38.01,504.08,1.89,5.15], \mathbf{u}_{3}^{*}=\left[0.0351,1.671,7.2557 e^{-4}, 0,0\right]$.
} 


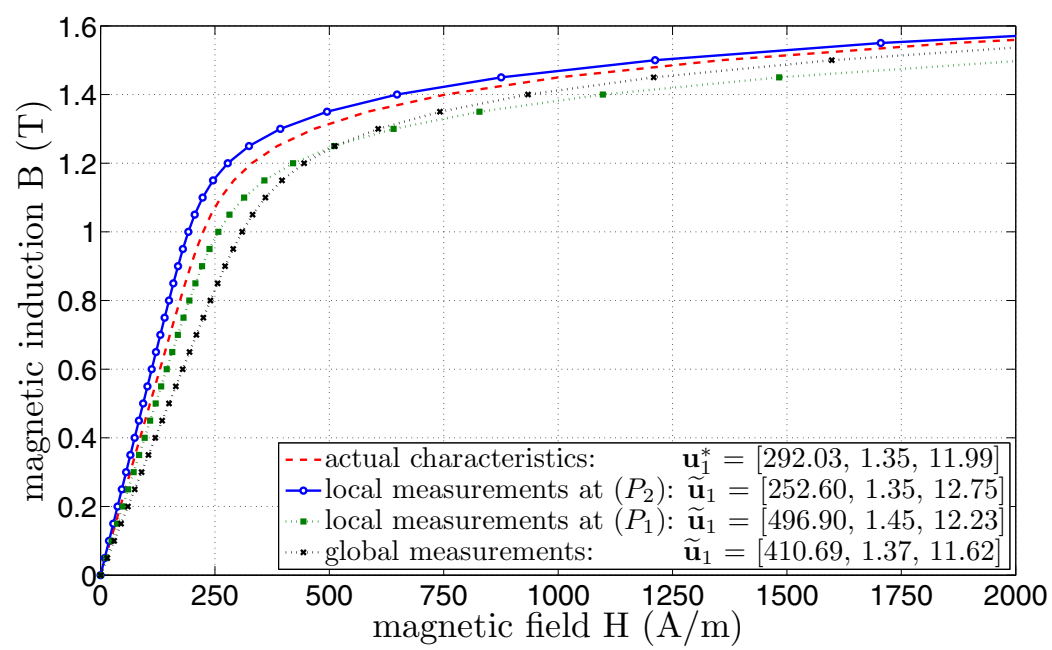

Fig. 4. Recovered $B-H$ characteristics based on real $B_{m}$ at $P_{2}$ and $P_{1}$ and $\phi_{m}$ compared to the actual characteristic, $\left(g_{2}=0\right)$.

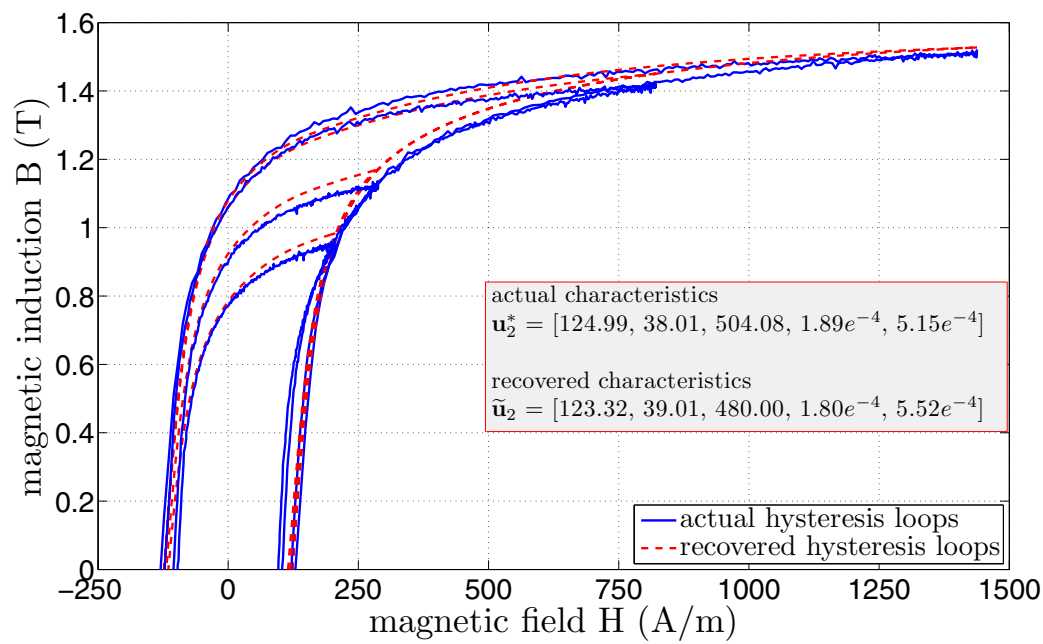

Fig. 5. The half of the hysteresis loops using $\Gamma_{2}$ compared to the actual hysteretic characteristic at $(f=1 \mathrm{~Hz}$.).

\subsubsection{Identification of the loss parameters}

After retrieving the values of $\widetilde{\mathbf{u}}_{1}$, an a posteriori inverse problem, based on (Equation 62) and (Equation 67), is solved for the identification of the loss parameters of the EI core material.

For simplicity, and due to the fact that the excess loss is absent in the studied material $c_{1}, d_{1}=$ 0 in (Equation 61), we restrict ourselves only to the identification of the first three parameters in $\mathbf{u}_{2}$. Fig. 6 shows the reconstructed loss characteristics of the EI core material, for different 


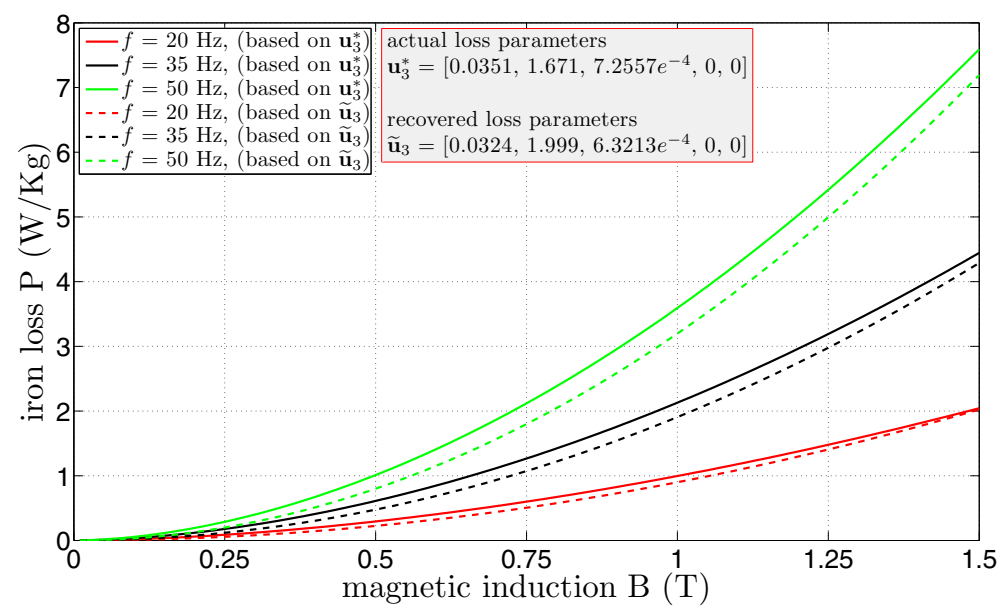

Fig. 6. The loss characteristics of the EI core material based on the reconstructed loss parameters compared to loss characteristics based on the original loss parameters.

frequency and magnetic induction levels, compared to the actual loss characteristics. Again, a good correspondence is observed. It is clear from Fig. 6 that the original grade of the EI core material at $1.5 \mathrm{~T}$ is $7.5 \mathrm{~W} / \mathrm{kg}$, however, the estimated one at $1.5 \mathrm{~T}$ is $7.15 \mathrm{~W} / \mathrm{kg}$.

\subsection{Error reduction}

In this section, the error originating from the uncertain geometrical model parameters is decreased by implementing the proposed MPU technique presented in section 7.1. Furthermore, a Bayesian approximation error approach, presented in section 7.2, is utilized for the error reduction when a coarse model is used in the inverse problem.

\subsubsection{MPU inverse problem formulation}

Due to the fact that $g_{2}$ is the most critical geometrical parameter, we use the MPU technique for reducing the error originating only form the uncertainty in the value of $g_{2}$. The traditional objective function $\Gamma_{1}$ is reformulated as follows:

$$
\Gamma_{1, M P U}(\mathbf{u})=\sum_{k=1}^{K}\left\|\mathbf{B}_{s}\left(\mathbf{u}, g_{2}\right)+\alpha\left(\frac{\partial \mathbf{B}_{s}\left(\mathbf{u}, g_{2}\right)}{\partial g_{2}}\right)-\mathbf{B}_{m}\right\|_{\left(g_{2}=g_{2}^{*}, \text { position } 2\right)}^{2}
$$

with constant $\alpha . g_{2}^{\bullet}$ is the value of $g_{2}$ used in the forward model. It is possible to make an approximation of the Taylor coefficient if many measurement samples are available so as to make a linear fitting between the vector $\left(\mathbf{B}_{m}-\mathbf{B}_{s}\left(\mathbf{u}, g_{2}\right)\right)$ and vector $\left(\frac{\partial \mathbf{B}_{s}\left(\mathbf{u}, g_{2}\right)}{\partial g_{2}}\right)$. When minimizing the objective function (Equation 69), a path (parameter values $\mathbf{u}^{(j)}$ for the $j^{\text {th }}$ iteration) is followed that is minimally affected by the uncertainties. Indeed, a linear forward model, i.e. $B_{S}\left(I_{k}, \mathbf{u}, g_{2}\right)+\alpha\left(\frac{\partial B_{s}\left(I_{k}, \mathbf{u}, g_{2}\right)}{\partial g_{2}}\right)$, is used with the incorporation of the dependence to the uncertain parameter values and with the estimate of the uncertainty $\left(g_{2}\right)$. 


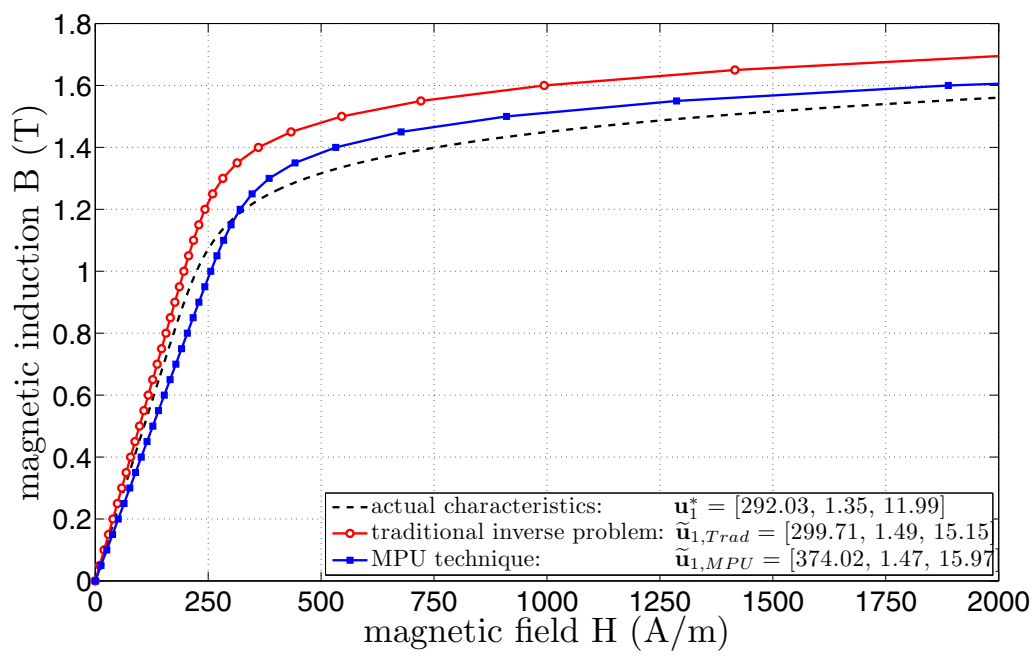

Fig. 7. The recovered $B-H$ curves based on the traditional and the MPU techniques compared to the original characteristics, for the experimental validation of the proposed technique on the EI core inductor application, $\left(g_{2}=0.25 \mathrm{~mm}\right)$.

In order to validate 'experimentally' the proposed MPU technique, two inverse problems (traditional and MPU) are solved starting from real measurements. The local magnetic induction is measured, at $P_{2}$, for $K=16$ sinusoidal excitation current values $I=$ $[0.5,1,1.5, \ldots, 8] \mathrm{A}$, and for $g_{2}=0.25 \mathrm{~mm}$. The value of $g_{2}$ is taken from the thickness of the inserted solid spacer between the E- and I-yokes. The inverse problems in the traditional and MPU formulation are solved yielding two recovered $B-H$ curves. These $B-H$ curve results are compared with the original $B-H$ curve. Fig. 7 depicts the recovered $B-H$ curves based on the traditional and the MPU techniques compared to the original characteristics, for $g_{2}=0.25$ $\mathrm{mm}$. It is clear from Fig. 7 that the MPU technique results in a more accurate recovered characteristics compared to the traditional inverse problem.

\subsubsection{Bayesian approximation error approach}

In all results presented above, we use a high fidelity model of the EI core, i.e. 3D finite element model with very fine mesh discretizations. However, this model is very time consuming. One may reduce the burden of the inverse problem by incorporating a relatively faster but not accurate coarse model. Using the Bayesian approximation error approach, presented in section 7.2, it is possible to reduce the error initiated from incorporating a coarse model instead of a fine model in the inverse problem.

For the sake of the comparison, we build three computer models: a very fine model based on 3D-FE with fine mesh discretizations 'model-a', a moderate fine model based on 3D-FE with coarse mesh discretizations 'model- $b$ ', and a coarse model based on a magnetic reluctance network 'model-c' in a similar way as presented in (Cale et al., 2006). Here, we consider 'model-a' as the fine model, however, the other two models, i.e. 'model- $b$ ' and 'model- $\mathrm{c}$ ', are considered as 'relatively' coarse models. 


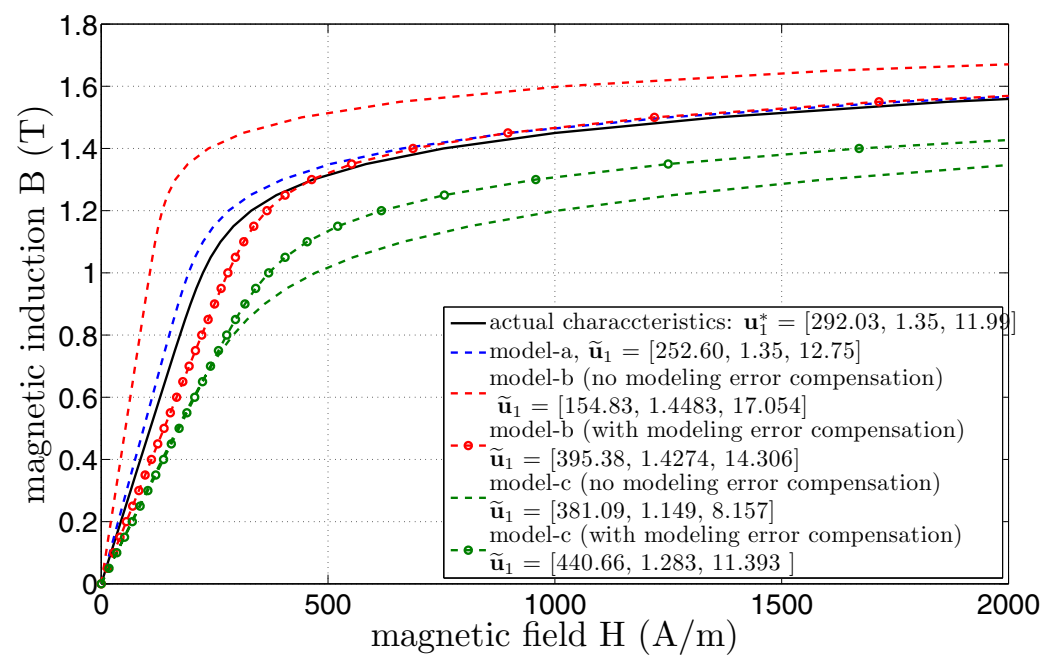

Fig. 8. The recovered $B-H$ curve using the two inverse problems based on the traditional Bayesian approach 'no modeling error compensation' and the Bayesian approximation error approach 'with modeling error compensation' for the models-b and $\mathrm{c}$, and the recovered characteristics for model-a, compared to the actual characteristics, $\left(g_{2}=0\right)$.

The value of $g_{2}$ is kept zero in order to eliminate the modeling uncertainty caused by the uncertain value of $g_{2}$, as previously explained. The local magnetic induction measurements at position $2\left(P_{2}\right)$ are used as being the input of the inverse problem.

In order to represent the modeling error between the fine and the coarse model stochastically, the procedure presented earlier in section 7.2.1 is used. $Z$ hypothetical values of $\mathbf{u}$ are generated using the Latin hypercube sampling technique (Viana et al., 2010). Z forward fine and coarse model computations are solved, and their responses 'local magnetic induction measurements' (i.e. $\mathbf{B}_{s}$ ) are compared.

Different inverse problems are solved, with the assumption ${ }^{11}$ of $\mathbf{e}_{n}=0$, for each computer model. Then, the identified magnetic characteristics (single-valued $B-H$ curve) are compared. For model-a, only one inverse problem is solved based on the traditional Bayesian approach, (Equation 56). However, for models-b and c, four inverse problems are solved, two for each computer model. The first inverse problem is based on the traditional Bayesian approach, (Equation 56), in which the modeling error is not compensated. While, the other inverse problem is based on the Bayesian approximation error approach, (Equation 58), in which the modeling error is compensated.

Fig. 8 illustrates the solution of the five inverse problems compared to the original characteristics. It is clear from Fig. 8 that the inverse problem based on (Equation 58) gives better results compared to the one based on (Equation 56) for both relatively coarse models. Of course, model-b results in a better solution compared to model-c due to the fact that model-c

11 This assumption is reasonable because the local magnetic induction measurements contain only a very limited amount of noise. In addition, this small measurement noise is difficult to quantify precisely in practice. 
is coarser than model-b. The results presented in this section validate 'experimentally' the proposed approach.

\section{General discussion}

In the previous sections, we applied the proposed inverse procedure for identifying the properties of the magnetic material inside an electromagnetic device (EI core electromagnetic inductor). The recovery error is 'qualitatively' estimated using the Cramér-Rao lower bound technique. Moreover, two different techniques are shown for a 'quantitative' reduction in the recovery error. The MPU technique 'iteratively' adapts the objective function to be minimized with respect to the uncertain model parameter. The Bayesian approximation error approach is used for compensating the modeling error originated from simplification of the mathematical model.

We are convinced that the stochastic Cramér-Rao bound method is the crucial part in the proposed methodology; it is fast, accurate and gives a better 'qualitative' view for the inverse problem results. Indeed, it neither depends on the fictitious parameter values, nor the accuracy of the used mathematical model. Moreover, it can be applied a priori (before solving the real inverse problem). The two other 'relatively time consuming' techniques, i.e. MPU and the Bayesian approximation error, are important for a 'quantitative' reduction of the recovery error. In fact, the three techniques, i.e. CRB, MPU and Bayesian, are not competitive techniques. Rather, they are integrative techniques. Also, it is worth mentioning that the 'true' values of the magnetic material properties are not needed in the identification procedure, we use it here only for validating the inverse procedure. All results presented using the three techniques are consistent.

In general, we think that the inverse problems based on local magnetic measurements are better than that based on global magnetic measurements or mechanical measurements, i.e. torque measurements in rotating electrical machines (Abdallh et al., 2011b). In practice, the local measurement should be carried out in regions with less stray fields, e.g. far from the excitation sources, sample edges and air gap (Abdallh \& Dupré, 2010b). Also, the sensors should be precisely positioned and calibrated (Abdallh \& Dupré, 2010c). The optimal number of sensors can be determined using the CRB technique, or using a special selection procedure, based on the presented MPU technique, such as the one presented in (Yitembe et al., 2011).

The most accurate knowledge about the model parameters increases the accuracy of the inverse problem. However, it is not essential to know precisely the values of the model parameters. MPU technique can be used for reducing the error of the uncertainties in these model parameters provided that the sensitivity analysis with respect to these model parameters can be calculated. Furthermore, coupling the measurements with the most accurate fine 'time demanding' model results in the best recovery solution. Again, the Bayesian approximation error approach can be used for reducing the modeling error when a 'time efficient' coarse model is incorporated in the inverse procedure, provided that a stochastic modeling error is existed.

Although we applied, in this chapter, the proposed methodology for the identification of the magnetic material inside relatively large scale industrial applications, such as the considered EI electromagnetic core inductor, the inverse procedure can be used also in small scale applications, such as the reconstruction of magnetic nanoparticles (Crevecoeur et al., 2012). 
Also, the inverse procedure can be used for nondestructive magnetic evaluation of defects in magnetic materials (Durand et al., 2006).

\section{Conclusions}

An efficient coupled experimental-numerical inverse problem is proposed for characterizing the properties of a magnetic material. The proposed methodology is capable of estimating a priori the error in the inverse problem solution. This a priori 'qualitative' error estimation takes into account the measurement noise and the uncertainties in the model parameters in a stochastic framework based on the Cramér-Rao lower bound technique. Moreover, a deterministic method based on the sensitivity analysis is presented for reducing 'quantitatively' the error due to the uncertainties in the model parameters. Furthermore, the stochastic Bayesian approach is adapted in order to reduce the error in the inverse problem solution present when a coarse model is used. The complete procedure of the presented inverse problem methodology ensures not only the optimum experimental design but also the best numerical scheme. The proposed methodology is applied for identifying the magnetic properties of a magnetic material inside an EI electromagnetic core. The methodology is tested numerically and validated experimentally. The obtained results reveal the success and the reliability of the proposed scheme, which can be used for any other application. Finally, the main advantage of the proposed procedure is that it offers an efficient and consistent framework for the magnetic material characterization using the coupled experimental-numerical inverse problem approach. To our knowledge, it is the first time to propose a complete inverse problem procedure for identifying the properties of magnetic materials. On the other hand, it is the case that the proposed methodology is much more time consuming compared to the direct identification techniques based only on measurement results. However, this is the 'cost' that is needed for the 'non-destructive' identification of a magnetic material inside a complex geometry. Further research will focus on solving stochastic inverse problems in order to identify more magnetic material properties.

\section{Acknowledgment}

The authors gratefully acknowledge the financial support of the projects FWO-G.0082.06 of the Fund of Scientific Research-Flanders, and GOA07/GOA/006 of the "Bijzonder Onderzoeksfonds" of Ghent University.

\section{References}

Abdallh, A.; Sergeant, P.; Crevecoeur, G.; Vandenbossche, L.; Dupré, L. \& Sablik, M. (2009). Magnetic material identification in geometries with non-uniform electromagnetic fields using global and local magnetic measurements, IEEE Transactions on Magnetics, Vol. 45, pp. 4157-4160.

Abdallh, A.; Crevecoeur, G. \& Dupré, L. (2010a). Optimal needle placement for the accurate magnetic material quantification based on uncertainty analysis in the inverse approach, Measurement Science and Technology, Vol. 21, pp. 115703(16pp).

Abdallh, A. \& Dupré, L. (2010b). A Rogowski-Chattock coil for local magnetic field measurements: sources of error, Measurement Science and Technology, Vol. 21, pp. 107003(5pp). 
Abdallh, A. \& Dupré, L. (2010c). Local magnetic measurements in magnetic circuits with highly non-uniform electromagnetic fields, Measurement Science and Technology, Vol. 21, pp. 045109(10pp).

Abdallh, A.; Sergeant, P.; Crevecoeur, G. \& Dupré, L. (2010d). An inverse approach for magnetic material characterization of an EI core electromagnetic inductor, IEEE Transactions on Magnetics, Vol. 46, pp. 622-625.

Abdallh, A.; Crevecoeur, G. \& Dupré, L. (2011a). A robust inverse approach for magnetic material characterization in electromagnetic devices with minimum influence of the air gap uncertainty, IEEE Transactions on Magnetics, Vol. 47, pp. 4364-4367.

Abdallh, A.; Crevecoeur, G. \& Dupré, L. (2011b). Selection of measurement modality for magnetic material characterization of an electromagnetic device using stochastic uncertainty analysis, IEEE Transactions on Magnetics, Vol. 47, pp. 4564-4573.

Abdallh, A.; Sergeant, P.; Crevecoeur, G. \& Dupré, L. (2011c). Magnetic material identification of a switched reluctance motor, International Journal of Applied Electromagnetics and Mechanics, Vol. 37, pp. 35-49.

Abdallh, A.; Crevecoeur, G. \& Dupré, L. (2012a). A priori experimental design for inverse identification of magnetic material properties of an electromagnetic device using uncertainty analysis, International Journal for Computation and Mathematics in Electrical and Electronic Engineering (COMPEL), Vol. 31, pp. 972-984.

Abdallh, A.; Sergeant, P. \& Dupré, L. (2012b). A non-destructive methodology for estimating the magnetic material properties of an asynchronous motor, IEEE Transactions on Magnetics, Vol. 48, No. 4, in press.

Abdallh, A.; Crevecoeur, G. \& Dupré, L. (2012c). A Bayesian approach for stochastic modeling error reduction of magnetic material identification of an electromagnetic device, Measurement Science and Technology, Vol. 23, pp. 035601(12pp).

Abdallh, A.; Crevecoeur, G. \& Dupré, L. (2012d). Impact reduction of the uncertain geometrical parameters on magnetic material identification of an EI electromagnetic inductor using an adaptive inverse algorithm, Journal of Magnetism and Magnetic Materials, Vol. 324, pp. 1353-1359.

Alifanov, O.; Artyukhin, E. \& Rumyantsev, S. (1995). Extreme methods for solving ill-posed problems with applications to inverse problems, Begell House, ISBN: 978-1567000382, Wallingford, UK.

Bandler, J.; Cheng, Q.; Dakroury, S.; Mohamed, A.; Bakr, M.; Madsen, K. \& S $\phi$ ndergaard, J. (2008). Space mapping: state of the art, IEEE Transactions Microwave Theory and Techniques, Vol. 52, pp. 337-361.

Beck, J. \& Woodbury, K. (1998). Inverse problems and parameter estimation: integration of measurements and analysis, Measurement Science and Technology, Vol. 9, pp. 839-847.

Bertotti, G. (1988). General properties of power losses in soft ferromagnetic materials, IEEE Transactions on Magnetics, Vol. 24, pp. 621-630.

Bertotti, G. (1998). Hysteresis in magnetism: for physicists, materials scientists, and engineers, Academic Press, ISBN: 978-012093270, San Diego, California, USA.

Cale, J.; Sudhoff, S. \& Turner, J. (2006). An improved magnetic characterization method for highly permeable materials, IEEE Transactions on Magnetics, Vol. 42, pp. 1974-1981.

Crevecoeur, G. (2009). Numerical methods for low frequency electromagnetic optimization and inverse problems using multi-level techniques, Ph.D. Thesis, Ghent University, Belgium. 
Crevecoeur, G.; Abdallh, A.; Couckuyt, I.; Dupré, L. \& Dhaene, T. (2011a). Two-level refined direct optimization scheme using intermediate surrogate models for electromagnetic optimization of a switched reluctance motor, Engineering with Computers, in press.

Crevecoeur, G.; Baumgarten,D.; Steinhoff, U.; Haueisen, J.; Trahms, L. \& Dupré, L. (2012). Advancements in magnetic nanoparticle reconstruction using sequential activation of excitation coil arrays using magnetorelaxometry, IEEE Transactions on Magnetics, in press.

Durand, S.; Cimrak, I.; Sergeant, P. \& Abdallh, A. (2010). Analysis of a non-destructive evaluation technique for defect characterization in magnetic materials using local magnetic measurements, Mathematical Problems in Engineering, article ID 574153 (19 pages).

Echeverría, D.; Lahaye, D.; Encica, L.; Lomonova, E.; Hemker, P. \& Vandenput, A. (2006). Manifold-Mapping optimization applied to linear actuator design, IEEE Transactions on Magnetics, Vol. 42, pp. 1183-1186.

Emery, A.; Nenarokomov, A. \& Fadale, T. (2000). Uncertainties in parameter estimation: The optimal experiment, International Journal of Heat and Mass Transfer, Vol. 43, pp. 3331-3339.

Emery, A.; Valenti, E. \& Bardot, D. (2007). Using Bayesian inference for parameter estimation when the system response and experimental conditions are measured with error and some variables are considered as nuisance variables, Measurement Science and Technology, Vol. 18, pp. 19-29.

Engl, H.; Hanke, M. \& Neubauer, A. (1996). Regularization of Inverse Problems (Mathematics and its Applications), Kluwer Academic Publishers, ISBN: 978-0792361404, Nederlands.

Fadale, T.; Nenarokomov, A. \& Emery, A. (1995a). Uncertainties in parameter estimation: The inverse problem, International Journal of Heat and Mass Transfer, Vol. 38, pp. 511-518.

Fadale, T.; Nenarokomov, A. \& Emery, A. (1995b). Two approaches to optimal sensor locations, Journal of Heat Transfer, Vol. 117, pp. 373-379.

Fiorillo, F. (2004). Measurement and characterization of magnetic materials, Academic Press, ISBN: 978-0122572517, San Diego, California, USA.

Gaignaire, R.; Crevecoeur, G.; Dupré, L.; Sabariego, R.; Dular, P. \& Geuzaine, C. (2010). Stochastic uncertainty quantification of the conductivity in EEG source analysis by using polynomial chaos decomposition, IEEE Transactions on Magnetics, Vol. 46, pp. 3457-3460.

Goodwin, G.; Zarrop, M. \& Payne, R. (1974). Coupled design of test signals, sampling intervals, and filters for system identification, IEEE Transactions on Automatic Control, Vol. 19, pp. 748-752.

Goodwin, G. \& Payne, R. (1977). Dynamic system identification. Experiment design and data analysis, Academic Press, ISBN: 978-0122897504, New York, USA.

International Electrotechnical Commission 'IEC' International Standard 404-2. (1996). Methods of measurement of the magnetic properties of electrical steel sheet and strip by means of an Epstein frame. Geneva, Switserland.

International Electrotechnical Commission 'IEC' International Standard 404-3. (2000). Methods of measurement of the magnetic properties of electrical steel sheet and strip by means of a single sheet tester. Geneva, Switserland.

Kaipio, J. \& Somersalo, E. (2005). Statistical and computational inverse problems (Applied Mathematical Sciences Vol. 160), Springer, ISBN: 978-0387220734, New York, USA. 
Lauwagie, T. (2003). Vibration-based methods for the identification of the elastic properties of layered materials, Ph.D. Thesis, Katholieke Universiteit Leuven, Belgium. http://www.mech.kuleuven.be/dept/resources/docs/lauwagie.pdf.

Leach, R.; Giusca, C. \& Naoi, K. (2009). Development and characterization of a new instrument for the traceable measurement of areal surface texture, Measurement Science and Technology, Vol. 20, pp. 125102(8pp).

Marquardt, D. (1963). An algorithm for least-squares estimation of nonlinear parameters, SIAM Journal on applied Mathematics, Vol. 11, pp. 431-441.

National Institute of Standards and Technology 'NIST' (2000). Essentials of expressing measurement uncertainty. http://physics.nist.gov/cuu/Uncertainty/index.html.

Nissinen, A; Heikkinen, L. \& Kaipio, J. (2008). The Bayesian approximation error approach for electrical impedance tomography - experimental results, Measurement Science and Technology, Vol. 19, pp. 015501(9pp).

Radich, B. \& Buckley, K. (1995). EEG dipole localization bounds and MAP algorithms for head models with parameter uncertainties, IEEE Transactions Biomedical Engineering, Vol. 42, pp. 233-241.

Saitoh, S. (2007). Applications of Tikhonov regularization to inverse problems using reproducing kernels, Journal of Physics: Conference Series, Vol. 73, ID. 012019.

Scales, J. \& Snieder, R. (2000). The anatomy of inverse problems, Geophysics, Vol. 65, pp. 1708-1710.

Sergeant, P.; Crevecoeur, G.; Dupré, L. \& Van den Bossche, A. (2009). Characterization and optimization of a permanent magnet synchronous machine, COMPEL: The International Journal for Computation and Mathematics in Electrical and Electronic Engineering, Vol. 28, pp. 272-285.

Sievert, J. (2000). The measurement of magnetic properties of electrical sheet steel - survey on methods and situation of standards, Journal of Magnetism and Magnetic Materials, Vol. 215, pp. 647-651.

Stoica, P. \& Nehorai, A. (1989). MUSIC, Maximum-likelihood, and Cramér-Rao bound, IEEE Transactions on Acoustics, Speech and Signal Processing, Vol. 37, pp. 720-774.

Strang, G. (1986). Introduction to applied mathematics, Wellesley, ISBN: 978-0961408800, Cambridge, UK.

Takahashi, N.; Morimoto, H.; Yunoki, Y. \& Miyagi, D. (2008). Effect of shrink fitting and cutting on iron loss of permanent magnet motor, Journal of Magnetism and Magnetic Materials, Vol. 320, pp. E925-E928.

Tarantola, A. (2004). Inverse problem theory and methods for model parameter estimation, Society for industrial and applied mathematics (SIAM), ISBN: 978-0898715729, Philadelphia, USA.

Viana, F.; Venter, G. \& Balabanov, V. (2004). An algorithm for fast optimal Latin hypercube design of experiments, International Journal for Numerical Methods in Engineering, Vol. 82 , pp. 135-156.

Yitembe, B.; Crevecoeur, G.; Van Keer, R. \& Dupré, L. (2011) Reduced conductivity dependence method for increase of dipole localization accuracy in the EEG inverse problem, IEEE Transactions Biomedical Engineering, Vol. 58, pp. 1430-1440. 


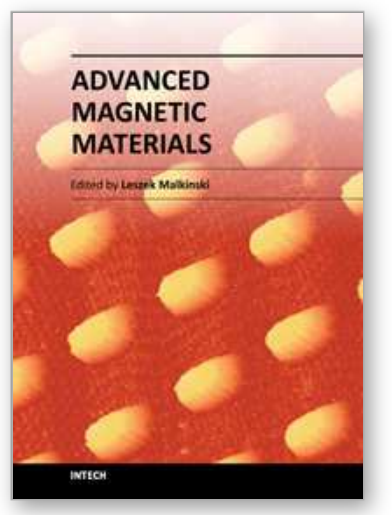

\author{
Advanced Magnetic Materials \\ Edited by Dr. Leszek Malkinski
}

ISBN 978-953-51-0637-1

Hard cover, 230 pages

Publisher InTech

Published online 24, May, 2012

Published in print edition May, 2012

This book reports on recent progress in emerging technologies, modern characterization methods, theory and applications of advanced magnetic materials. It covers broad spectrum of topics: technology and characterization of rapidly quenched nanowires for information technology; fabrication and properties of hexagonal ferrite films for microwave communication; surface reconstruction of magnetite for spintronics; synthesis of multiferroic composites for novel biomedical applications, optimization of electroplated inductors for microelectronic devices; theory of magnetism of Fe-Al alloys; and two advanced analytical approaches for modeling of magnetic materials using Everett integral and the inverse problem approach. This book is addressed to a diverse group of readers with general background in physics or materials science, but it can also benefit specialists in the field of magnetic materials.

\title{
How to reference
}

In order to correctly reference this scholarly work, feel free to copy and paste the following:

Ahmed Abouelyazied Abdallh and Luc Dupré (2012). Magnetic Material Characterization Using an Inverse Problem Approach, Advanced Magnetic Materials, Dr. Leszek Malkinski (Ed.), ISBN: 978-953-51-0637-1, InTech, Available from: http://www.intechopen.com/books/advanced-magnetic-materials/magnetic-materialcharacterization-using-an-inverse-problem-approach

\section{INTECH}

open science | open minds

\author{
InTech Europe \\ University Campus STeP Ri \\ Slavka Krautzeka 83/A \\ 51000 Rijeka, Croatia \\ Phone: +385 (51) 770447 \\ Fax: +385 (51) 686166 \\ www.intechopen.com
}

\author{
InTech China \\ Unit 405, Office Block, Hotel Equatorial Shanghai \\ No.65, Yan An Road (West), Shanghai, 200040, China \\ 中国上海市延安西路65号上海国际贵都大饭店办公楼405单元 \\ Phone: +86-21-62489820 \\ Fax: $+86-21-62489821$
}


(C) 2012 The Author(s). Licensee IntechOpen. This is an open access article distributed under the terms of the Creative Commons Attribution 3.0 License, which permits unrestricted use, distribution, and reproduction in any medium, provided the original work is properly cited. 